\title{
Out-of-Plane Constraint Loss in Three Point Bend Specimens with Notches
}

\author{
A J Horn ${ }^{1 *}, \mathrm{~S} \mathrm{Cicero}^{2}$, D Andrés ${ }^{3 \dagger}$
}

1. Wood Nuclear, 305 Bridgewater Place, Birchwood Park, Risley, Cheshire. WA3 6XG. UK.

2. LADICIM (Laboratory of Materials Science and Engineering), University of Cantabria, E.T.S. de Ingenieros de Caminos, Canales y Puertos, Av/ Los Castros 44, 39005, Santander, Cantabria, Spain.

3. Technology Department, UK Atomic Energy Authority, Culham Science Centre, Abingdon, Oxfordshire. OX14 3DB UK.

* Corresponding Author, email: Anthony.Horn@woodplc.com

$\dagger$ Address at the time of performing the work: LADICIM (Laboratory of Materials Science and Engineering), University of Cantabria, E.T.S. de Ingenieros de Caminos, Canales y

Puertos, Av/ Los Castros 44, 39005, Santander, Cantabria, Spain

(C) 2020. This manuscript version is made available under the CC-BY-NC-ND 4.0 license http://creativecommons.org/licenses/by-nc-nd/4.0/ 


\begin{abstract}
This paper presents an experimental and numerical study of the effect of specimen thickness on the effective notch toughness $K_{\text {mat }}^{\rho}$ for cleavage fracture measured using Single Edge Notch Bend (SENB) specimens containing a U-notch instead of a fatigue pre-crack. These specimens are typically used to measure a material's effective notch toughness $K_{\text {mat }}^{\rho}$ and to assess failure of a structure containing a non-sharp defect using the Notch Failure Assessment Diagram (NFAD). Both the experimental data and the Finite Element (FE) failure predictions show a significant influence of specimen thickness on $K_{m a t}^{\rho}$, over and above the microstructural weakest link effect arising from differences in the volume of the plastic zone. $K_{\text {mat }}{ }^{\rho}$ is a function of not only the in-plane effect of the notch radius, but also an out-of-plane constraint loss which itself is enhanced by the presence of the notch radius. Significant out-of-plane constraint loss occurred for notched specimens with a ratio of thickness $B$ to width $W$ of 0.5 , a geometry that if pre-cracked would have met the minimum thickness requirement mandated by ASTM E1820. Doubling the thickness to $B / W=1.0$ was sufficient to eliminate the out-of-plane constraint loss. The use of experimentally measured $K_{\text {mat }}^{\rho}$ values in an NFAD assessment of a structure may therefore be non-conservative if $B / W<1.0$.
\end{abstract}

KEYWORDS: Blunt notch, effective fracture toughness, Notch Failure Assessment Diagram.

\title{
NOMENCLATURE
}

a

$B$

E

$e_{\max }$

$f\left(L_{r}\right)$

$f\left(L_{r}^{\rho}\right)$

$i$

$J$

$J^{\rho}$

$J_{E S I S}$

$K_{I}$

$K_{I}^{\rho}$

$K_{J}$

$K_{j}^{\rho}$

$K \rho_{C}$

$K_{\text {mat }}$

$K_{\text {mat }}^{\rho}$

$K_{\text {min }}$

$K_{r}$

$K_{r}^{\rho}$

$L$

$L_{r}$

$L_{r}^{\rho}$

$L_{r(\max )}$

$l$

m

$n$

N

$r$

P

Crack or notch depth (from the notch mouth to the tip of the notch)

Specimen thickness

Elastic modulus

Strain at maximum load

Failure assessment curve on the FAD

Failure assessment curve on the NFAD

ith toughness value in a dataset (out of a total of $N$ values)

Elastic-plastic energy release rate

$J$ for a notch

$J$ obtained from load-displacement data using ESIS P2-92, Equation 7

Linear elastic stress intensity factor

Linear elastic stress intensity factor for a notch

$J$ expressed in dimensions of $K$

$J^{\rho}$ expressed in dimensions of $K$

Critical value of $K_{j}^{\rho}$ for an individual test specimen containing a notch

$K_{J C}$ measured using pre-cracked specimens at a defined $P_{f}$

$K_{J C}$ measured using notched specimens at a defined $P_{f}$

Minimum possible value of $K_{\text {mat }}$, defined as $20 \mathrm{MPa} \sqrt{\mathrm{m}}$ in ASTM E1921

Fracture ratio, plotted on ordinate axis of the FAD

Fracture ratio for a notch, plotted on ordinate axis of the NFAD

Element size

Load ratio, plotted on the abscissa axis of the FAD

Load ratio for a notch, plotted on abscissa axis of the NFAD

Maximum value of $L_{r}$ defining vertical cut-off on the FAD

Material parameter describing sensitivity of $K_{\text {mat }}^{\rho} / K_{\text {mat }}$ to $\sigma_{N} / \sigma_{y}$

Weibull modulus

Strain hardening exponent

Total number of toughness values in a dataset

Distance from the centre of curvature of the notch root radius

Applied load 


\begin{tabular}{|c|c|}
\hline$P_{f}$ & Failure probability \\
\hline$P_{L}$ & Limit load \\
\hline$P_{L}^{\rho}$ & Limit load for a notch \\
\hline$R$ & Radius of boundary layer model \\
\hline$R(m)$ & Error function defined in Section 4.3.1 \\
\hline$T$ & Temperature \\
\hline$T_{0}$ & Master Curve Reference Temperature \\
\hline$u_{1}, u_{2}$ & Displacements in the $x_{1}$ and $x_{2}$ directions respectively \\
\hline$V$ & Plastic zone \\
\hline$V_{0}$ & Reference volume taken as unity \\
\hline$W$ & Specimen width \\
\hline$x_{1}, x_{2}, x_{3}$ & Co-ordinate system \\
\hline Y & Geometry factor used to define $K_{I}$ \\
\hline$\gamma$ & Material parameter describing sensitivity of $K_{\text {mat }}^{\rho} / K_{m a t}$ to $\sigma_{N} / \sigma_{y}$ \\
\hline$\eta$ & Proportionality constant to evaluate $J$ from load vs. displacement \\
\hline$v$ & Poisson's ratio \\
\hline$\xi^{A, B}$ & Estimate of the SSY scale factor \\
\hline$\rho$ & Notch root radius \\
\hline$\theta$ & Angle subtended at the centre of curvature of the notch root radius \\
\hline$\sigma$ & Applied tensile stress \\
\hline $\bar{\sigma}$ & Flow stress, defined as the mean of $\sigma_{y}$ and UTS \\
\hline$\sigma_{1}$ & Maximum principal stress \\
\hline$\sigma_{N}$ & Elastic notch tip opening stress \\
\hline$\sigma_{0}$ & Stress at the limit of proportionality \\
\hline$\sigma_{u}$ & Weibull parameter, defined as $\sigma_{w}$ at $P_{f}=0.632$ \\
\hline$\sigma_{w}$ & Weibull stress \\
\hline$\sigma_{w, \min }$ & Minimum value of Weibull stress in SSY corresponding to $K_{\min }=20 \mathrm{MPa} \sqrt{\mathrm{m}}$ \\
\hline$\sigma_{y}$ & Yield stress defined at $0.2 \%$ plastic strain \\
\hline CMOD & Crack Mouth Opening Displacement \\
\hline CT & Compact Tension \\
\hline FAD & Failure Assessment Diagram \\
\hline FE & Finite Element \\
\hline LLD & Load-Line Displacement \\
\hline NFAD & Notch Failure Assessment Diagram \\
\hline SSY & Small Scale Yielding \\
\hline $\mathrm{SSY}^{\mathrm{N}}$ & Small Scale Yielding for a notch \\
\hline UTS & Ultimate Tensile Stress \\
\hline
\end{tabular}




\section{INTRODUCTION}

The structural integrity of engineering structures is conventionally assessed using defect or flaw assessment procedures based on fracture mechanics approaches [e.g. 1-3]. For a real or postulated defect, the crack driving force (e.g. the elastic-plastic energy release rate $J$ or the elastic-plastic stress intensity factor $K_{J}$ ) under the loading conditions and temperature of interest is compared with the material fracture toughness. Such procedures assume flaws to be infinitely sharp. While this assumption may be appropriate for fatigue cracks, in other cases such as porosity, lack-of-fusion, corrosion damage, mechanical damage, or even design features such a crevices in tube-to-tubeplate assemblies, it can be an over-conservative assumption that can lead to a pessimistic assessment of the structure and a significant under-estimation of the safety margin against fracture.

Structural integrity assessments undertaken in accordance with [1-3] are carried out using a Failure Assessment Diagram (FAD) in which the ordinate $K_{r}$ indicates the proximity to fracture. For primary loading only, $K_{r}$ is defined as $K_{I} / K_{\text {mat }}$, where $K_{I}$ is the linear elastic stress intensity factor and $K_{m a t}$ is the material toughness typically derived from fatigue pre-cracked fracture toughness specimens tested according to well-defined standards, e.g. [4-6]. The abscissa $L_{r}$ indicates the proximity to failure by plastic collapse and is defined as $P / P_{L}$, where $P$ is the applied load and $P_{L}$ is the elastic-perfectly plastic limit load. $K_{r}$ and $L_{r}$ are both proportional to $P$ and a linear loading line can be plotted on the FAD. When all inputs are best-estimate values, failure is predicted at its intersection with the failure assessment curve which is represented by $K_{r}=f\left(L_{r}\right)$ for $L_{r}<L_{r(\max )}$ where $f\left(L_{r}\right)$ is the failure assessment curve, $L_{r(\max )}$ is the ratio of the uniaxial flow stress $\bar{\sigma}$ to the uniaxial yield stress $\sigma_{y}$ defined at $0.2 \%$ plastic strain, and $\bar{\sigma}$ is defined as the mean of $\sigma_{y}$ and the ultimate tensile stress (UTS).

Over the last 25 years or so, several engineering assessment methodologies have been published in the literature for assessing structures that contain non-sharp defects using a modified form of the FAD called the Notch Failure Assessment Diagram (NFAD) [e.g. 7-12]. The exact form of the NFAD varies from approach to approach. Taking the approach described in [7] and [12] as an example, proximity to the two failure limits of plastic collapse and fracture is quantified by the parameters $L_{r}^{\rho}$ and $K_{r}^{\rho}$. $L_{r}^{\rho}$ is defined as $P / P_{L}^{\rho}$, where $P_{L}^{\rho}$ is the elastic-perfectly plastic limit load for a component containing a notch of root radius $\rho . K_{r}^{\rho}$ is defined as $K_{I}^{\rho} / K_{\text {mat }}^{\rho}$, where $K_{I}^{\rho}$ is the linear-elastic notch stress intensity factor and $K_{m a t}^{\rho}$ is the effective notch toughness. The condition that the component does not fail is indicated by $K_{r}^{\rho}<f\left(L_{r}^{\rho}\right)$ for $L_{r}^{\rho_{r}}<L_{r(\max )}$. Several authors [7, 12-14] have shown that when the NFAD axes are defined by $L_{r}^{\rho}$ and $K_{r}^{\rho}$ instead of $L_{r}$ and $K_{r}$, failure assessment curves are broadly independent of $\rho$. This enables the same failure assessment curve to be used in the NFAD as for the FAD.

Although the precise definitions of the parameters used in the various forms of NFAD vary between the different approaches, one similarity common to all NFAD approaches is the requirement to use an effective notch toughness $K_{m a t}^{\rho}$ in place of the material toughness $K_{\text {mat }}$, to calculate $K_{r}^{\rho}$. No testing standards are currently available to provide guidance on how $K_{\text {mat }}{ }^{\rho}$ can be measured using fracture toughness specimens that contain notches instead of pre-cracks. In the absence of dedicated test standards for notched specimens, test standards originally designed for pre-cracked specimens such as [4-6] have been used widely in the literature [e.g. 7, 11, 17-22] to obtain values of $K_{\text {mat }}^{\rho}$ for notched specimens. It has recently been shown $[15,16]$ that in most cases, such testing standards can provide reasonably accurate values of $K_{\text {mat }}^{\rho}$ for notched specimens.

More recent work [23] has shown that the values of $K_{\text {mat }}^{\rho}$ measured on laboratory specimens containing notches are not only dependent on the in-plane effect of the notch radius, but are also significantly affected by an out-of-plane constraint loss which is itself enhanced by the presence of the notch radius. The effect of out-of-plane constraint loss is an active research topic for sharp cracks 
$[24,25]$. For notches this out-of-plane constraint loss can have a greater effect on toughness than that of the in-plane effect of the notch radius alone. The use of experimentally measured $K_{\text {mat }}{ }^{\rho}$ values in an NFAD assessment of a notched structure may therefore be non-conservative if the out-of-plane constraint loss in the test specimen is more significant than that in the structure. The work in [23] was based solely on mechanistic modelling, and to the authors' knowledge there are no experimental data available for notched specimens to confirm the conclusions where one thickness is compared with another. The objective of the work presented in this paper is to demonstrate experimentally whether $K_{m a t}^{\rho}$ values for cleavage fracture are dependent on specimen thickness, and whether the effect can be successfully described using mechanistic modelling. 


\section{BACKGROUND}

The NFAD approaches in [7-12] describe the increase in effective notch toughness either as a function of $\rho$, or another parameter that characterises the notch radius. The approach described in [12] relates $K_{m a t}^{\rho}$ to the component of the elastic notch tip stress $\sigma_{N}$ acting in a direction perpendicular to the plane of the notch. $\sigma_{N}$ scales with load: a given value of $\sigma_{N}$ could correspond to an acute notch under low load, or a blunter notch subject to a higher load. An expression for $\sigma_{N}$ was derived by Shin [27] based on the Creager-Paris elastic stress distribution ahead of a slender notch in a uniform stress field [28]:

$$
\sigma_{N}=\sigma\left(1+2 Y \sqrt{\frac{a}{\rho}}\right)
$$

where $\sigma$ is the applied tensile stress remote from the notch and $Y$ is a geometry factor used to define $K_{I}$ via $K_{I}=Y \sigma \sqrt{\pi a}$.

The following empirical power law expression was found to describe the increase in effective cleavage toughness with increasing notch radius in [12], and preliminary work in [29] indicated that the same expression may be used to describe the increase in ductile tearing initiation toughness with notch radius:

$$
\frac{K_{m a t}^{\rho}}{K_{m a t}}=1+\gamma\left[\frac{\sigma_{N}}{\sigma_{0}}\right]^{-l}
$$

where $\gamma$ and $l$ are non-dimensional material parameters that describe the sensitivity of material toughness to the notch root radius. $\sigma_{N}$ can be normalised using any convenient parameter, in Equation 2 it is normalised by $\sigma_{0}$, the yield stress defined as the stress at the limit of proportionality. Equation 2 defines the failure locus shown in Figure 1. A loading line may be plotted on the diagram for a notched component of interest, with failure being predicted by its intersection with the failure locus. For the loading lines, the vertical axis is defined as $K_{J}^{\rho} / K_{\text {mat }}$ where $K_{J}^{\rho}$ is $J$ for a notch, $J^{\rho}$, expressed in dimensions of $K . K_{J}^{\rho}$ can be obtained from either the $J$-integral, or the area under the load vs. displacement curve, using the methods described in Section 4.1. For blunt notches the loading curve rises steeply and failure is predicted at high $K_{\text {mat }}^{\rho}$ values, and for acute notches the loading curve is less steep and failure is predicted at lower $K_{\text {mat }}^{\rho}$ values. As $\rho \rightarrow 0$, the gradient of the failure locus at its intersection with the loading line approaches the horizontal and $K_{\text {mat }}^{\rho} \rightarrow K_{\text {mat }}$.

Depending on failure mechanism, values of $\gamma$ and $l$ that define the failure locus in Figure $1 \mathrm{can}$ be obtained using one of several methods:

(a) For initiation by cleavage and ductile tearing, curve fitting to test data plotted in the form of $K \rho_{C} / K_{\text {mat }}$ vs. ${ }_{N} / \sigma_{0}$ can be performed, where $K \rho_{C}$ is the value of $K_{J}^{\rho}$ at failure for an individual test specimen containing a notch. This is straightforward for cleavage fracture, but is less so for ductile tearing initiation due to the lack of test guidance and the practical challenges of measuring and defining tearing initiation from a notch tip.

(b) For initiation by cleavage only, the lookup table presented in [12] can be used based on a knowledge of Weibull modulus $m$ and strain hardening exponent $n$.

(c) For initiation by cleavage only, a combination of (a) and (b) can be used, useful for when $m$ is unknown but $n$ is known.

(d) For initiation by cleavage and ductile tearing, micromechanical modelling using an appropriate local approach failure criterion can be used. 


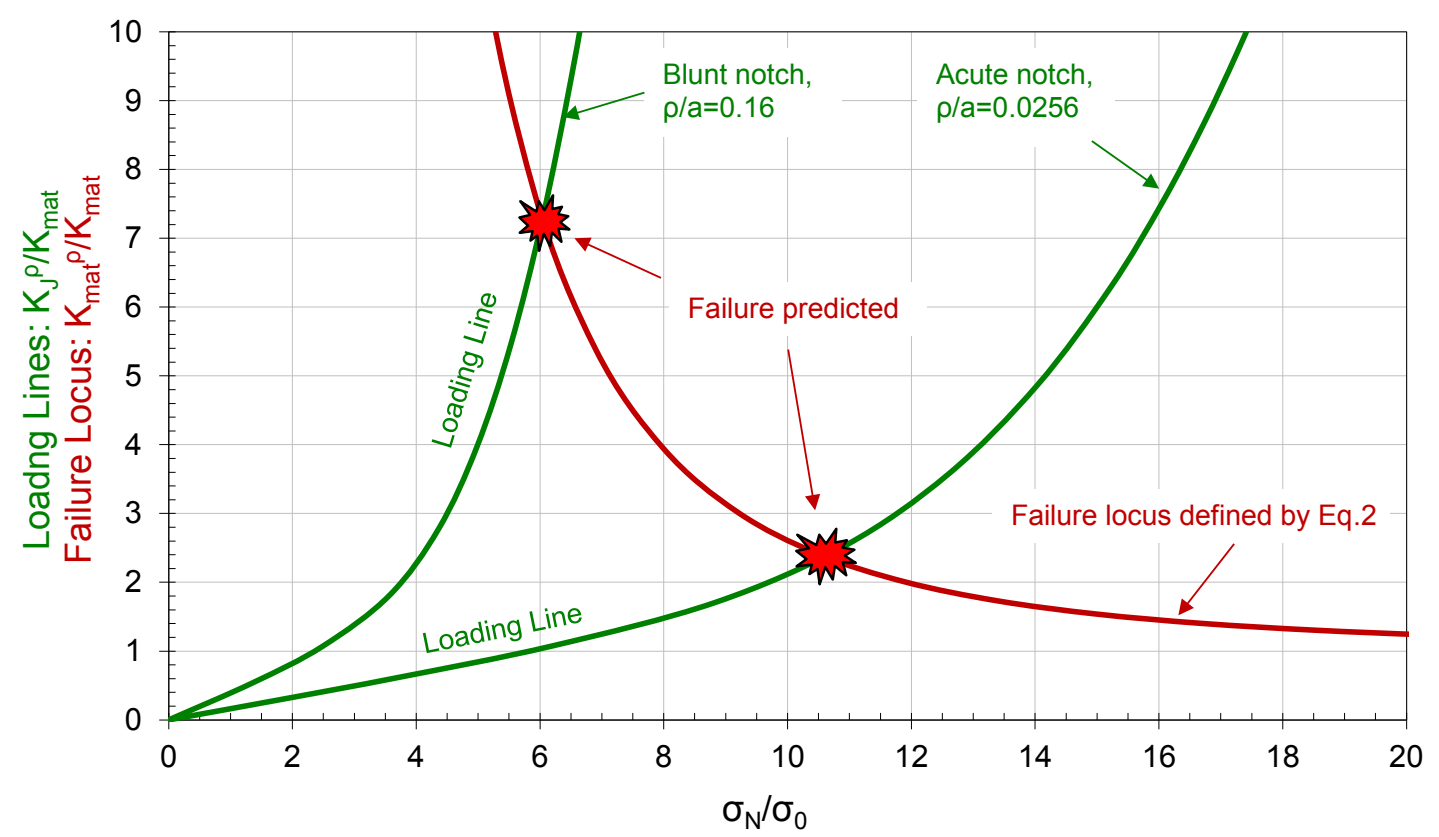

Figure 1: Loading lines and failure locus in toughness- $\sigma_{N}$ space

The lookup table for use in option (b) requires a knowledge of the Weibull modulus $m$, a parameter used in the Beremin model [30] which describes the proximity to cleavage fracture by use of the scalar Weibull stress, $\sigma_{w}$. In its simplest form the probability of fracture $P_{f}$ is described by a twoparameter Weibull distribution:

$$
P_{f}=1-\exp \left[-\left(\frac{\sigma_{w}}{\sigma_{u}}\right)^{m}\right]
$$

where $\sigma_{u}$ and $m$ are the Weibull parameters. $m$ is the shape parameter describing the scatter, and $\sigma_{u}$ is the scale parameter, defined as the value of $\sigma_{w}$ at $P_{f}=0.632$. The Weibull stress is calculated by integrating a weighted value of the maximum principal stress $\sigma_{1}$ over the plastic zone $V$ ahead of the stress concentration:

$$
\sigma_{w}=\left[\frac{1}{V_{0}} \int_{V} \sigma_{1}^{m} d V\right]^{1 / m}
$$

The constant $V_{0}$ is a reference volume required to ensure dimensional consistency and in the current work is taken as unity. The Weibull parameters $m$ and $\sigma_{u}$ are determined by matching values calculated from the Beremin model to experimental values of cleavage fracture toughness. Reliable estimation of the Weibull parameters is only possible using experimental data of sufficient quantity that cover two different constraint levels. The method proposed by Gao et al [31] is suitable. A detailed description of the method and its application to the current work is described later in Section 4.3.1 of this paper.

One of the useful aspects of this approach, as shown in [12], is that for a given value of $\sigma_{N} / \sigma_{0}$, the ratio $K_{\text {mat }}^{\rho} / K_{\text {mat }}$ remains independent of load, independent of $J$, independent of $\sigma_{w}$, and independent of cleavage fracture probability $P_{f}$. The cleavage fracture probability is introduced into the approach 
when a value is assigned to $K_{\text {mat }}$. For example, a $K_{\text {mat }}$ value corresponding to a $5 \%$ cleavage fracture probability would enable $K_{\text {mat }}{ }^{2}$ at that same cleavage fracture probability to be defined.

\section{MATERIAL}

The material selected for the experimental programme was a $15 \mathrm{~mm}$ thick structural steel plate of grade S460M [32] which has been used previously for studying notch effects on fracture [33,34]. Table 1 summarises the chemical composition of the material, performed by means of chemical emission spectroscopy, and Figure 2 shows the microstructure, which comprises alternate bands of pearlite and ferrite.

Table 1. Chemical composition of steel S460M.

\begin{tabular}{|l|c|c|c|c|c|c|c|c|c|c|c|c|c|}
\hline & $\mathbf{C}$ & $\mathbf{S i}$ & $\mathbf{M n}$ & $\mathbf{P}$ & $\mathbf{S}$ & $\mathbf{C r}$ & $\mathbf{M o}$ & $\mathbf{N i}$ & $\mathbf{A l}$ & $\mathbf{C u}$ & $\mathbf{N b}$ & $\mathbf{T i}$ & $\mathbf{V}$ \\
\hline $\mathbf{S 4 6 0}$ & 0.1 & 0.4 & 1.4 & 0.01 & 0.00 & 0.06 & 0.00 & 0.01 & 0.04 & 0.01 & 0.03 & 0.00 & 0.06 \\
$\mathbf{M}$ & 2 & 5 & 9 & 2 & 1 & 2 & 1 & 6 & 8 & 1 & 6 & 3 & 6 \\
\hline
\end{tabular}

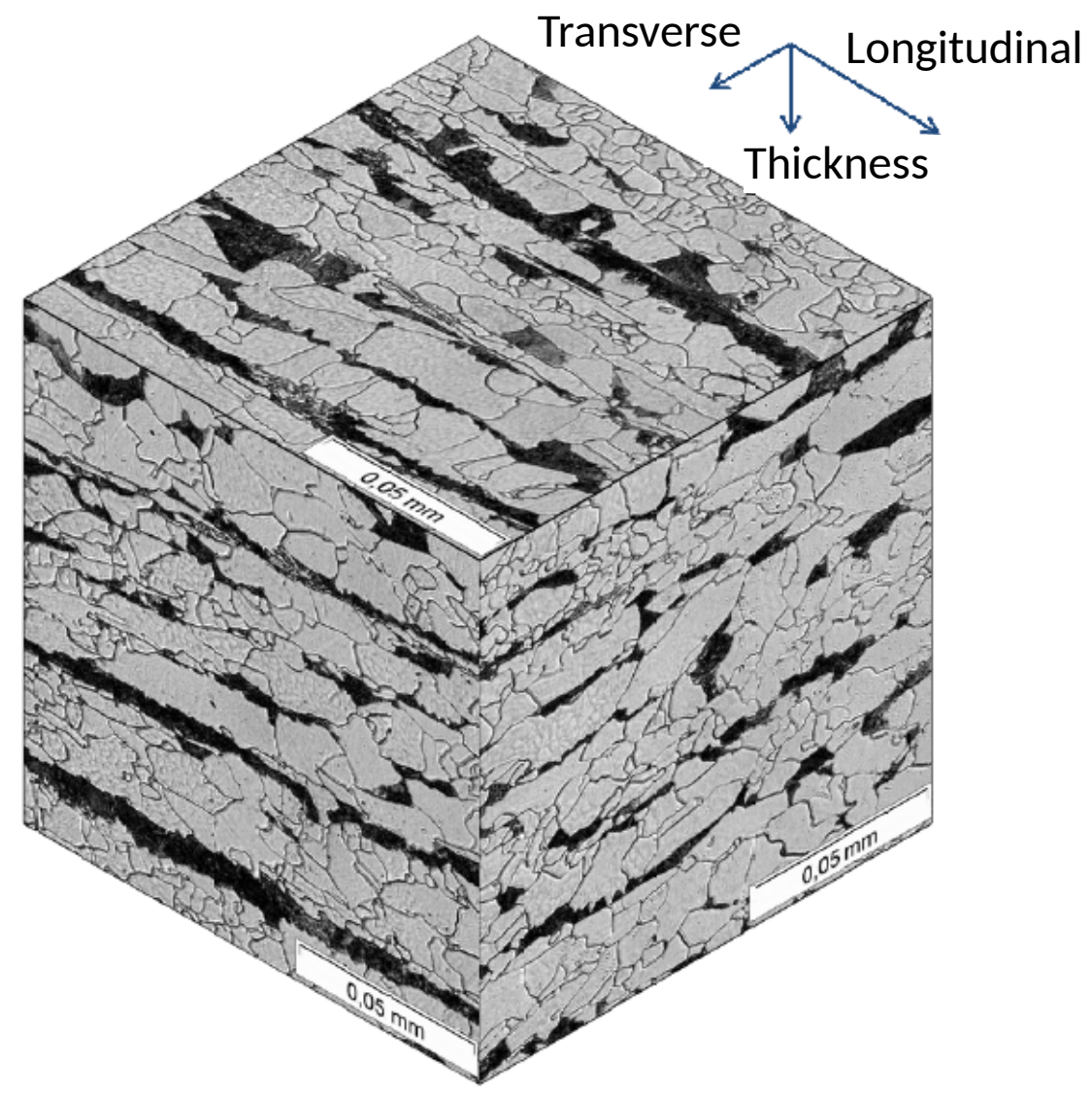

Figure 2: Microstructure of steel S460M, with ferritic-pearlic microstructure (sample polished and etched with Nital $2 \%$ ).

The elastic modulus $E$ was defined using the following expression due to Ingham et al [36]:

$$
E=210000(M P a)-54 T
$$


where $T$ is the temperature in ${ }^{\circ} \mathrm{C}$. The value of $E$ at the test temperature of $-100^{\circ} \mathrm{C}$ was therefore taken as $215400 \mathrm{MPa}$. Poisson's ratio $v$ was assumed to be 0.3 .

Tensile properties were measured using cylindrical tensile specimens $10 \mathrm{~mm}$ in diameter and machined from the centre of the plate thickness, parallel to the rolling direction (i.e. longitudinal). Four repeat tensile tests were performed at $-100^{\circ} \mathrm{C}$ in accordance with ASTM E8 [35]. Tensile curves were found to be discontinuous, exhibiting yield plateaus prior to strain hardening. Table 2 summarises the tensile properties $\sigma_{y}$ (defined at $0.2 \%$ plastic strain), Ultimate Tensile Strength (UTS), and the strain at maximum load $e_{\max }$.

Table 2: Tensile properties of steel $\mathrm{S} 460 \mathrm{M}$ at $-100{ }^{\circ} \mathrm{C}$.

\begin{tabular}{|c|c|c|c|c|}
\hline & Test No. & $\sigma_{y}$ (MPa) & UTS (MPa) & $e_{\max }(\mathbf{\%})$ \\
\hline \multirow{4}{*}{ S460M } & 1 & 632.0 & 724.5 & 12.0 \\
\cline { 2 - 5 } & 2 & 590.1 & 719.7 & 14.2 \\
\cline { 2 - 5 } & 3 & 622.8 & 722.4 & 11.5 \\
\cline { 2 - 5 } & 4 & 618.2 & 710.8 & 14.2 \\
\cline { 2 - 5 } & Average values & 615.7 & 719.3 & 12.9 \\
\hline
\end{tabular}

Fracture toughness properties of the same plate were determined in previous work $[33,34]$ where the Master Curve Reference Temperature, $\mathrm{T}_{0}[37,38]$, was calculated as $-91.8^{\circ} \mathrm{C}$. The fracture toughness $K_{\text {mat }}$ as defined using the Master Curve approach includes a crack front length correction to account for the microstructural weakest link effect in pre-cracked specimens. For a given $T_{0}, K_{m a t}$ for any specimen thickness $B$, cleavage fracture probability $P_{f}$ and temperature $T$ within the transition region is defined as follows:

$$
K_{\text {mat }}=20(M P a)+\left[\ln \left(\frac{1}{1-P_{f}}\right)\right]^{1 / 4}\left\{11+77 \exp \left[0.019\left(T-T_{0}\right)\right]\right\}\left(\frac{25}{B}\right)^{1 / 4}
$$

The experimental programme focused on two specimen thicknesses, the full plate thickness of $B$ $=15 \mathrm{~mm}$, and $B=9 \mathrm{~mm}$. This latter thickness was selected as the thinnest possible specimen that, if precracked, would still meet the minimum thickness criterion required to ensure plane strain conditions as defined in [39]. This is discussed further in Section 6 below. For this material's $T_{0}$ value of $-91.8^{\circ} \mathrm{C}$, the median fracture toughness $K_{\text {mat }}$ at the chosen test temperature of $T=-100^{\circ} \mathrm{C}$ is $110.6 \mathrm{MPa} \sqrt{\mathrm{m}}$ for $B$ $=9 \mathrm{~mm}$ and $99.7 \mathrm{MPa} \sqrt{\mathrm{m}}$ for $B=15 \mathrm{~mm}$. A median fracture toughness was used for convenience for comparing with test data. 


\section{METHODOLOGY}

\subsection{Experimental Programme}

The experimental fracture programme reported in this paper comprises 24 Single Edge Notch Bend (SENB) specimens containing notches instead of fatigue pre-cracks. This does not include the precracked specimens used to determine $T_{0}$, reported in [33,34], or the notched specimens from the same papers. All specimens were machined from the same $15 \mathrm{~mm}$ thick plate from which the tensile specimens were machined, oriented parallel to the rolling direction, and notched in the throughthickness direction. As for the tensile tests, the fracture tests were performed at $-100^{\circ} \mathrm{C}$, just below the $\mathrm{T}_{0}$ value of $-91.8^{\circ} \mathrm{C}$. U-shaped notches were machined into the specimens using Electro-Discharge Machining (EDM) and the fracture tests were performed in accordance with ASTM E1820 [39]. In order to achieve the required test temperature, liquid nitrogen was used in combination with an insulating chamber.

The work reported in [33,34] did not consider the effect of thickness $B$ on effective notch toughness $K_{m a t}{ }^{\rho}$. As the aim of the current work is to compare test results from specimens of two different thicknesses $B$ but with all other geometrical dimensions kept the same, 24 new tests have been specifically performed for this work, with the geometrical dimensions summarised in Table 3. Six repeat tests were performed for each of the four geometries. The specimens with $\mathrm{B}=9 \mathrm{~mm}$ were machined from the plate centreline. Figure 3 shows the geometry of one type of specimen, D11-D16.

Table 3: Test Matrix with Geometrical Dimensions

\begin{tabular}{|l|l|l|l|l|l|l|l|l|l|}
\hline Codes & Description & $\begin{array}{l}\text { Specimen } \\
\text { Type }\end{array}$ & $\begin{array}{l}\text { B } \\
(\mathrm{mm})\end{array}$ & $\begin{array}{l}\mathrm{W} \\
(\mathrm{mm})\end{array}$ & $\begin{array}{l}\mathrm{a} \\
(\mathrm{mm})\end{array}$ & $\begin{array}{l}\rho \\
(\mathrm{mm})\end{array}$ & $\mathrm{B} / \mathrm{W}$ & $\mathrm{a} / \mathrm{W}$ & $\rho / \mathrm{a}$ \\
\hline D11-D16 & Thin Acute & SENB & 9 & 18 & 9 & 0.15 & 0.5 & 0.5 & 0.017 \\
\hline D21-D26 & Thin Blunt & SENB & 9 & 18 & 9 & 1.20 & 0.5 & 0.5 & 0.133 \\
\hline E11-E16 & Thick Acute & SENB & 15 & 18 & 9 & 0.15 & 0.833 & 0.5 & 0.017 \\
\hline E21-E26 & Thick Blunt & SENB & 15 & 18 & 9 & 1.20 & 0.833 & 0.5 & 0.133 \\
\hline
\end{tabular}
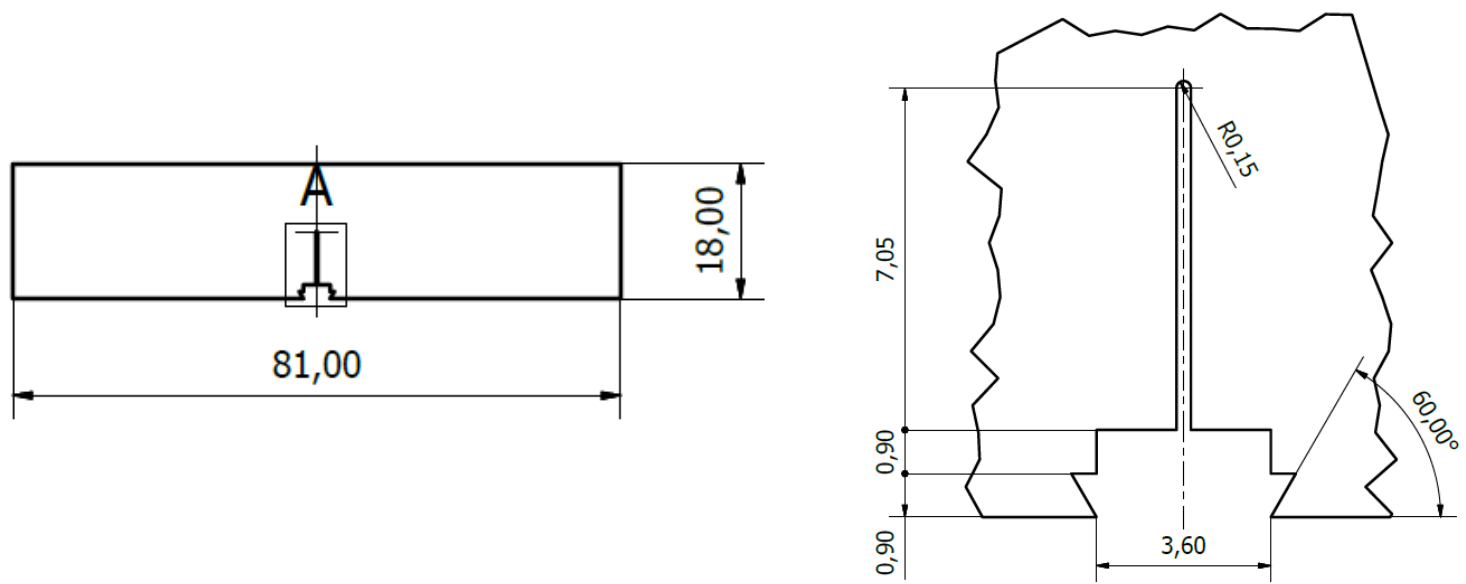

Figure 3: SENB fracture specimens D11 to D16 (see Table 3). Dimensions in mm.

Table 3 shows that the experimental program combines two different thicknesses $B$ (9mm and $15 \mathrm{~mm}$ ) and notch radii $\rho(0.15 \mathrm{~mm}$ and $1.2 \mathrm{~mm})$, but all other dimensions are kept constant. During the different tests, the applied load, the Crack Mouth Opening Displacement (CMOD) and the crosshead displacement were recorded. For some tests, the clip gauges measuring CMOD reached their maximum opening shortly before the end of the test, so in these cases CMOD for the final portion of the test was 
estimated. This was achieved by extrapolating the CMOD vs. time trend recorded during the midportion of the test. This trend was very close to being linear, but a $2^{\text {nd }}$ order polynomial fit provided a more accurate fit, and this was used to extrapolate CMOD values up to the end of the test.

Load Line Displacement (LLD) was not measured in the tests, but estimates of LLD were obtained using correlations between CMOD and LLD obtained from the Finite Element (FE) models reported in the next section below. Although the relationship between CMOD and LLD in each FE model was approximately linear and approximately the same for all four geometries shown in Table 3, a second order polynomial fitted to each individual geometry provided the most accurate relationship, and these polynomial expressions were used to estimate LLD from the CMOD measured in each test.

Although the tests were performed in accordance with the ASTM procedure, ESIS P2-92 [40] was used to calculate $J$, denoted here as $J_{E S I S}$, from the area under the load vs. LLD curve using

Equation 7:

$$
J_{E S I S}=\frac{\eta U}{B(W-a)}
$$

where $\eta=2$

$U=$ area under load vs. LLD curve.

Although Equation 7 was derived for use with pre-cracked specimens, as is the case for similar expressions in other fracture toughness testing standards, it has been shown $[12,15,16,29]$ that such expressions provide reasonable estimates of $J^{\rho}$, typically to within $10 \%$ depending on notch radius and loading level. Of all these methods, $J_{E S I S}$ generally provides the most accurate method for notched specimens, typically to within $5 \%[12,15]$.

Values of $J_{E S I S}$ at failure were converted to dimensions of $K$ using the following expression:

$$
K \rho_{C}=\sqrt{E J_{E S I S} /\left(1-v^{2}\right)}
$$

To characterise the improvement in effective toughness due to the presence of the notch, it is convenient to normalise the $K \rho_{C}$ measured for each notched specimen by the fracture toughness $K_{\text {mat }}$, where $K_{\text {mat }}$ is defined for a pre-cracked specimen of the same specimen thickness $B$ as that of the notched specimen. A different absolute value of $K_{\text {mat }}$ is therefore used for each specimen thickness as described in Section 3 of this paper. Defined in this way, the ratio $K \rho_{C} / K_{\text {mat }}$ compares the measured notch toughness with the equivalent fracture toughness of a pre-cracked specimen of the same thickness.

\subsection{Finite Element Analysis}

\subsubsection{SENB Specimens}

Three-dimensional (3D) FE models of the four SENB test specimen geometries were constructed and validated against test data. In addition to the four test specimen geometries listed in Table 3, four additional geometries were modelled corresponding to specimen thicknesses of $\mathrm{B}=18 \mathrm{~mm}$ and $27 \mathrm{~mm}$, corresponding to $\mathrm{B} / \mathrm{W}$ ratios of 1.0 and 1.5 respectively. The complete FE model matrix is shown in Table 4. For each SENB specimen modelled, symmetry conditions were specified along the uncracked ligament $\left(x_{2}=0\right)$ and the longitudinal mid-plane $\left(x_{3}=\mathrm{B} / 2\right)$ thereby enabling one quarter of 
each SENB specimen to be modelled numerically. An example of the one-quarter model of the SENB specimen with $\mathrm{B}=15 \mathrm{~mm}$ and $\rho=1.20 \mathrm{~mm}$ is shown in Figure 4. Each model consisted of quadratic 20 noded reduced integration hexahedral elements (C3D20R) arranged into 14 variable thickness layers. The thickest element layer was defined at the longitudinal mid-plane with thinner elements defined near the free surface to accommodate the reduced constraint approaching plane stress conditions. Each model had a straight notch front. The FE analyses were performed using ABAQUS version 6.14-3 [41] using a finite strain formulation.

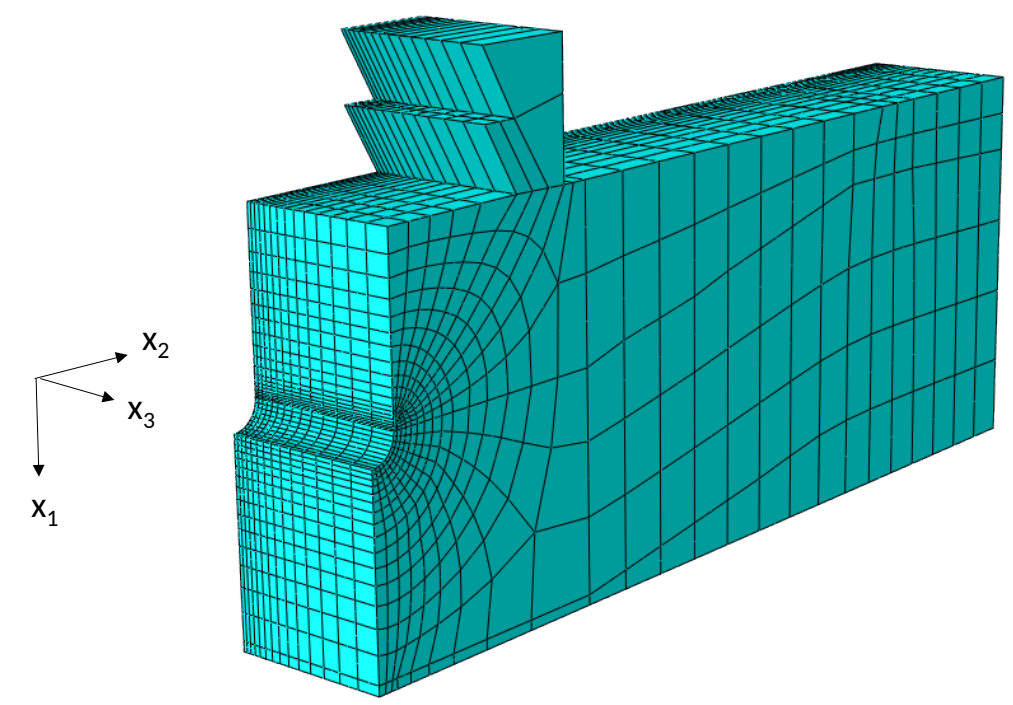

Figure 4: One-quarter FE model of SENB specimen E21-E26 (B=15mm, $\rho=1.20 \mathrm{~mm})$.

Table 4: FE Matrix

\begin{tabular}{|l|l|l|l|l|l|l|l|l|}
\hline \multirow{2}{*}{ Description } & $\begin{array}{l}\text { Specimen } \\
\text { Type }\end{array}$ & $\begin{array}{l}\text { B } \\
(\mathrm{mm})\end{array}$ & $\begin{array}{l}\mathrm{W} \\
(\mathrm{mm})\end{array}$ & $\begin{array}{l}\mathrm{a} \\
(\mathrm{mm})\end{array}$ & $\begin{array}{l}\rho \\
(\mathrm{mm})\end{array}$ & $\mathrm{B} / \mathrm{W}$ & $\mathrm{a} / \mathrm{W}$ & $\rho / \mathrm{a}$ \\
\hline \multirow{4}{*}{ Geometry matched to test data. } & SENB & 9 & 18 & 9 & 0.15 & 0.5 & 0.5 & 0.017 \\
\cline { 2 - 10 } & SENB & 9 & 18 & 9 & 1.20 & 0.5 & 0.5 & 0.133 \\
\cline { 2 - 10 } & SENB & 15 & 18 & 9 & 0.15 & 0.833 & 0.5 & 0.017 \\
\cline { 2 - 10 } & SENB & 15 & 18 & 9 & 1.20 & 0.833 & 0.5 & 0.133 \\
\hline \multirow{3}{*}{$\begin{array}{l}\text { No corresponding test data. } \\
\text { Geometry modelled to investigate } \\
\text { behaviour at higher thicknesses. }\end{array}$} & SENB & 18 & 18 & 9 & 0.15 & 1.0 & 0.5 & 0.017 \\
\cline { 2 - 10 } & SENB & 18 & 18 & 9 & 1.20 & 1.0 & 0.5 & 0.133 \\
\cline { 2 - 10 } & SENB & 27 & 18 & 9 & 0.15 & 1.5 & 0.5 & 0.017 \\
\cline { 2 - 9 } & SENB & 27 & 18 & 9 & 1.20 & 1.5 & 0.5 & 0.133 \\
\hline
\end{tabular}

Within each of the 14 variable thickness layers, rings of elements enclosed the notch tip as shown in Figure 5. The notch tip elements had a dimension $L$ in the $x_{1}$ direction and a dimension approximately equal to $L$ in the angular direction, $\theta=\tan ^{-1}\left(x_{2} / x_{1}\right)$. In the angular direction, 10 equally sized elements were defined in the range $0<\theta<\pi / 2$ and $L$ was constant with $\theta$. In the $x_{1}$ direction, $L$ increased with increasing distance $r$ from the centre of curvature of the notch tip, where $L=2 \pi r / 40$. The ratio $\rho / L$ was therefore the same in all models and ensured a consistency of mesh structures between the models with notches of differing radii. 


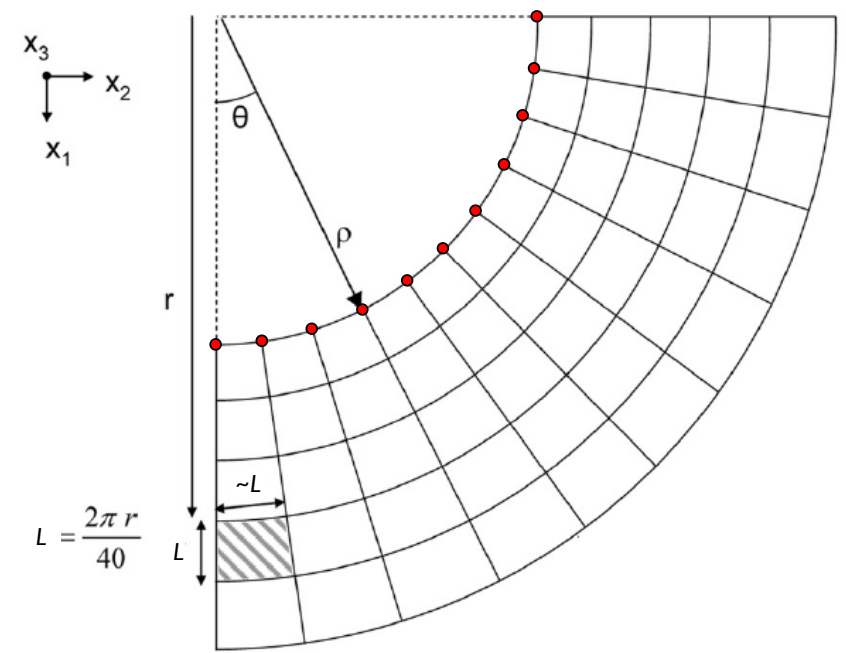

Figure 5: Notch tip mesh detail.

The lower loading pin was modelled using a three-dimensional rigid analytical part in the shape of a cylinder positioned below the SENB specimen. Loading in three-point bending was simulated by applying a prescribed displacement in the $x_{2}$ direction to a single reference point tied to a line of nodes on the top of the specimen. This enabled the reaction force to be evaluated through the single reference point.

Plasticity was modelled using true-stress vs. true-strain data obtained from the tensile tests performed at $-100^{\circ} \mathrm{C}$, and hence included the yield plateau as measured in the tensile tests. To obtain strains higher than those measured in the tensile tests, a Ramberg-Osgood relationship was used to extrapolate the test data to high strains. The stress at the limit of proportionality $\sigma_{0}$ was $597 \mathrm{MPa}$ and the strain hardening exponent $n$ used to extrapolate the stress-strain curve to high strains was 12 .

\subsubsection{Boundary Layer Models}

The method used for calibrating the Weibull parameters, described in Section 4.3.1 below, requires the use of a plane strain boundary layer FE model to simulate a crack in an infinite body [42]. The boundary layer model consisted of a semi-circular mesh of initial radius $R$ containing a radial crack modelled with a crack tip radius $\rho=2.5 \mu \mathrm{m}$. The ratio $R / \rho$ was set at $10^{5}$ to ensure that the crack tip plastic zone did not approach the boundary of the model thereby ensuring small-scale yielding conditions were preserved. Symmetry conditions were specified along the uncracked ligament $\left(x_{2}=0\right)$. Plane strain boundary conditions were applied to both faces in the $x_{3}$ direction, so the model was essentially 2D despite the same 3D elements being used as for the SENB specimens, i.e. quadratic 20noded reduced integration elements. Although the boundary layer model is essentially a plane strain analysis, the thickness of the model affects the volume of the crack tip plastic zone and hence the Weibull stress as defined by Equation 4. Four boundary layer models were analysed, each model having a thickness equal to that of each SENB model.

Displacement boundary conditions were applied incrementally to the nodes on the outer edge of the model. These displacements were consistent with the leading, $K_{I}$-dominated term of the Williams expansion [43] for the displacement field at the crack tip, as follows: 


$$
\begin{aligned}
& u_{1}=K_{I} \frac{1+v}{E} \sqrt{\frac{R}{2 \pi}} \cos \left(\frac{\theta}{2}\right)(3-4 v-\cos \theta) \\
& u_{2}=K_{I} \frac{1+v}{E} \sqrt{\frac{R}{2 \pi}} \sin \left(\frac{\theta}{2}\right)(3-4 v-\cos \theta)
\end{aligned}
$$

where $u_{1}$ and $u_{2}$ are the displacements in the $x_{1}$ and $x_{2}$ directions respectively, $E$ is the elastic modulus, $v$ is Poisson's ratio, and the polar co-ordinates $R$ and $\theta$ define the position of the node with respect to the crack tip. Plasticity was modelled using the same stress-strain relationship used for the SENB models.

\subsection{Post-processing}

\subsubsection{Calibration of Weibull parameter, $\mathrm{m}$}

The approach proposed by Gao et al [31] provides a suitable methodology for determining $m$ and $\sigma_{u}$ and can be summarised in the following steps:

1. Test two sets of fracture toughness specimens, one set corresponding to high constraint conditions (geometry A) and the other to low constraint conditions (geometry B).

2. Perform 3D elastic-plastic FE analyses of both specimen geometries tested (A and B). The models should have sufficient mesh refinement to allow accurate calculation of the Weibull stress $\sigma_{w}$ and the crack driving force $J$.

3. Perform 2D plane strain elastic-plastic FE analysis of a defect in an infinite body under SSY conditions using a boundary layer model.

4. Calibrate $m$ as follows:

a. Assume an $m$ value (or several trial values of $m$ ) and calculate the $\sigma_{w}$ vs. $J$ history for the A and B specimen geometries and for the SSY analysis

b. Constraint correct each measured $J$ value from the A and B specimen geometries to its equivalent SSY equivalent value. This is defined as the value of $J$ under small scale yielding which has the same scalar Weibull stress (and therefore failure probability) as the measured values of $J$.

c. Calculate two estimates of the SSY scale factor for the two distributions of constraintcorrected $J$ values. For $N$ toughness values, a simple estimate is given by:

$$
\xi^{A, B}=\left[\frac{1}{N}\left(\sum_{i=1}^{N} J_{(i)}{ }^{2}-S S Y\right)\right]^{1 / 2}
$$

d. Repeat steps (a-c) with different values of $m$ until $\xi^{A}=\xi^{B}$ within a small tolerance, thereby minimising the error function $R(m)=\left(\xi^{A}-\xi^{B}\right) / \xi^{B}$.

5. For the calibrated value of $m$, the value of $\sigma_{u}$ is the value of $\sigma_{w}$ in the boundary layer model corresponding to a crack driving force of $\xi^{A}=\xi^{B}$. 
The above method has previously been implemented for SENB specimens containing notches in [26] where it was noted that care is required during calibration to ensure that the constraint states of the high constraint geometry A and the low constraint geometry B span the defect of interest. This ensures that the model interpolates between the constraint states used for calibration, rather than extrapolating outside the range of applicability. For this reason, for Step 1, the thick specimen with an acute notch (E11-E16 in Table 3) was selected as the high constraint geometry A, and the thin specimen with a blunt notch (D21-D26) as the low constraint geometry B. This was to ensure the scaling model is applicable over the widest range of constraint states.

The calibration approach adopted uses SENB specimens of two different thicknesses, and for this reason Step 3 involved boundary layer analysis of the same two thicknesses as the SENB test specimens used for calibration. The constraint correction procedure in Step $4 \mathrm{~b}$ therefore corrected each specimen geometry to the equivalent SSY value for the boundary layer model of the same thickness, as shown schematically in Figure 6. In Step 5, one value of $\sigma_{u}$ was obtained for each thickness.

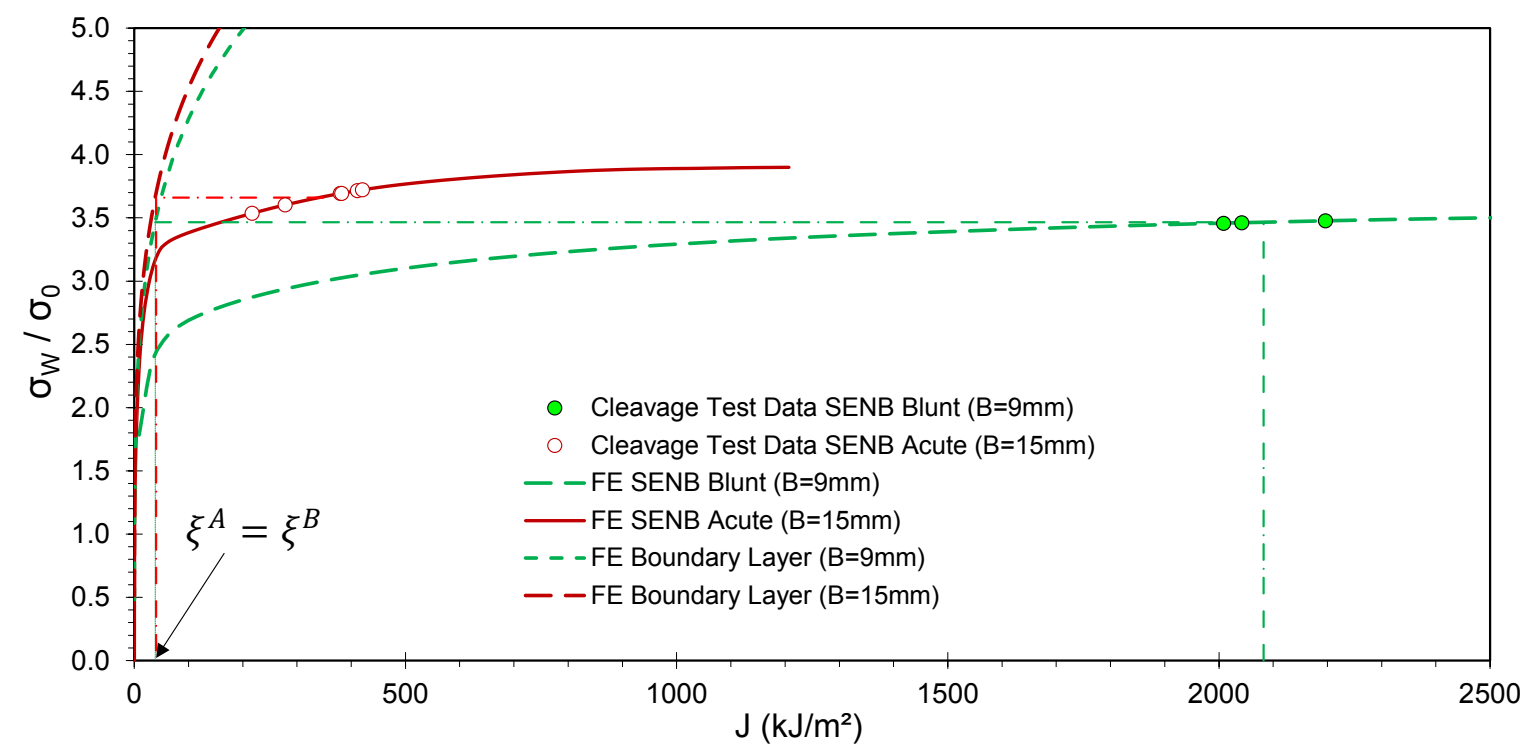

Figure 6: Toughness scaling diagram.

Equation 3 describes the probability of cleavage initiation only and does not account for subsequent micro-crack arrest (i.e. it assumes that cleavage initiation corresponds to macroscopic cleavage fracture). The two-parameter Weibull distribution therefore tends to over-predict the observed scatter in fracture toughness test results. This limitation led to an alternative expression being proposed [44] based on a three-parameter distribution:

$$
P_{f}=1-\exp \left[-\left(\frac{\sigma_{w}-\sigma_{w, \min }}{\sigma_{u}-\sigma_{w, \min }}\right)^{m}\right]
$$

where $\sigma_{w, \min }$ is the minimum value of Weibull stress at which macroscopic cleavage fracture becomes possible. $\sigma_{w, \min }$ is conventionally defined as the $\sigma_{w}$ value in SSY that corresponds to the lowest possible $K_{J}$ value at fracture, $K_{\text {min }}$, of $20 \mathrm{MPa} ل_{\mathrm{m}}$ as specified in ASTM E1921 [38]. For specimens with notches instead of fatigue pre-cracks, experimental data from steel specimens tested at very low temperatures (for example at $-196^{\circ} \mathrm{C}$ in [18]) indicate that even on the lower shelf, the 
measured effective notch toughness is significantly higher than the fracture toughness, which suggests that $K_{\min }$ may be larger than $20 \mathrm{MPa} / \mathrm{m}$ for specimens with notches. In this work, values of $\sigma_{w, \min }$ corresponding to a higher value of $K_{\text {min }}$, arbitrarily set at $50 \mathrm{MPa} \sqrt{\mathrm{m}}$, were considered in addition to the standard value of $20 \mathrm{MPa} \sqrt{\mathrm{m}}$ quoted for pre-cracked specimens in ASTM E1921.

\subsubsection{Weibull stress based toughness scaling model}

The toughness scaling model based on the Weibull stress [45] was originally developed for constraint correction between cracked specimens of differing constraint levels with different levels of applied $\mathbf{J}$ but identical cleavage fracture probabilities. The same approach was first applied to specimens containing cracks and notches of differing root radius in [26], and a similar approach has been adopted in this work.

Toughness scaling diagrams, such as those shown in Figure 6, were generated by post-processing numerical data (maximum principal stresses and integration point volumes) from each FE model to define the evolution of $\sigma_{w}$, calculated using Equation 4, with the value of $J$. For the SENB specimens, $J_{E S I S}$ was calculated according to ESIS P2-92 using Equation 7, for consistency with the analysis of the test data. For the boundary layer models representing SSY, $J$ cannot be obtained from Equation 7 so $J$ was instead obtained using contour independent $J$-integrals.

The probability of cleavage fracture is directly related to the Weibull stress via Equation 3. For specimens of the same thickness and therefore the same value for $\sigma_{u}$, a horizontal line plotted in Figure 6 defines a specific cleavage fracture probability. Figure 6 can then be used to predict the value of $J$ that corresponds to a given failure probability for any other geometry modelled of the same thickness. For specimens of different thickness and hence different values of $\sigma_{u}$, the toughness scaling model approach can still be used, but it is more convenient to plot $P_{f}$ instead of $\sigma_{w}$ on the ordinate axis to account for the different value of $\sigma_{u}$ for each specimen thickness. 


\section{RESULTS}

\subsection{Experimental Data}

Figure 7 plots the experimentally measured load vs. LLD curves for the SENB specimens together with the corresponding curves from the FE analyses. The FE analyses are discussed later in the paper. Most of the specimens failed in a brittle manner during a rising load. Examination of the fracture surfaces indicated failure occurred predominantly by cleavage fracture without significant ductile tearing, depicted by the circles in Figure 7. As an example, the fracture surface for specimen D26 is shown in Figure 8; despite extensive plastic deformation being apparent near the free surface, no significant pre-cleavage ductile tearing was visible on the fracture surface. In contrast, some of the other thinner specimens with $B / W=0.5$ did not fail by cleavage before maximum load was reached, and for these specimens the point of maximum load is represented by squares in Figure 7. For two specimens, problems during the test led to the test being stopped before fracture or maximum load was reached, these are shown by diamonds in Figure 7.
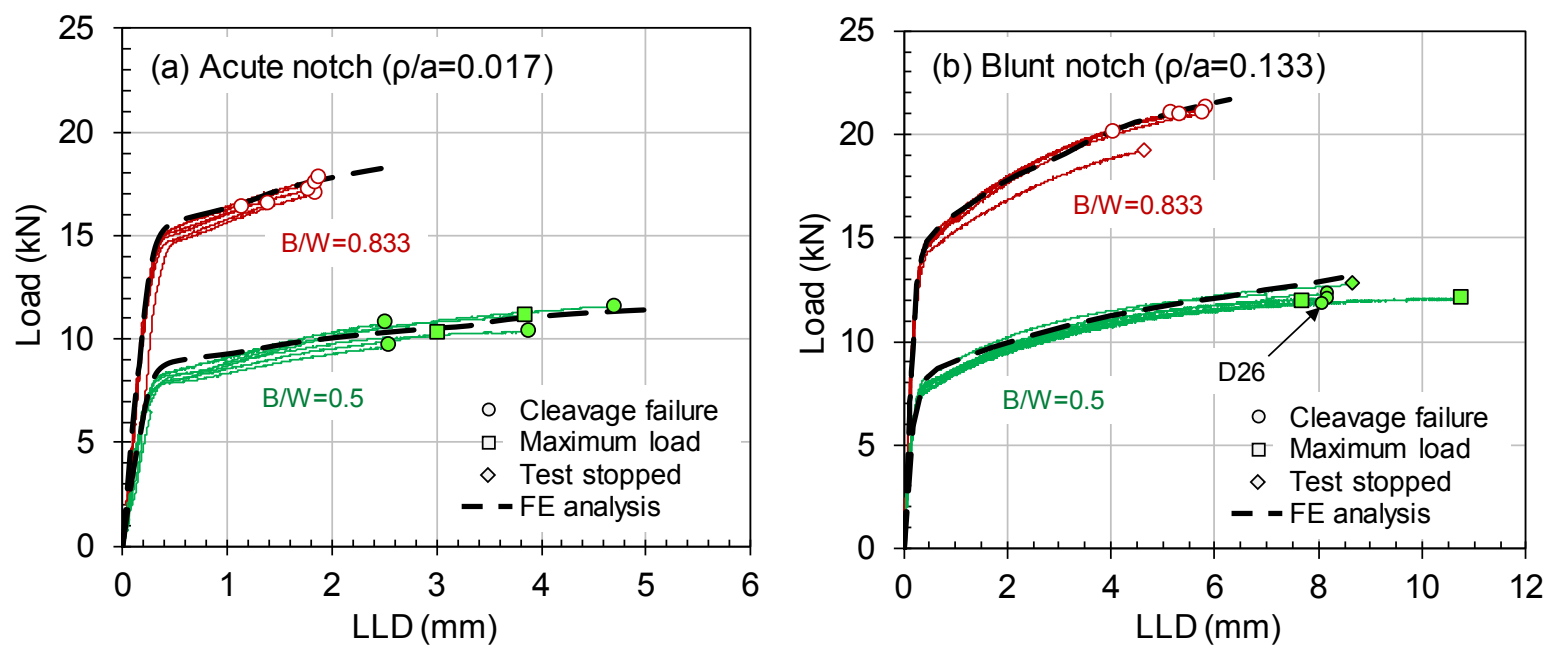

Figure 7: Load vs. displacement curves from the experimental programme showing specimens with (a) an acute notch and (b) a blunt notch.

From Figure 7 it is evident that the thicker specimens exhibited much higher loads than the thinner specimens, as would be expected due to the larger cross sectional area and hence greater load bearing capacity, and they also exhibited lower displacement values at failure than the thinner specimens. In terms of fracture mechanics, it is more useful to discuss the results in terms of the measured effective toughness, and this was calculated from the area under load vs. displacement curve using $J_{E S I S}$ (Equation 7) and converting to $K \rho_{C}$ (Equation 8). These values are summarised in Table 5 which tabulates the failure type, load at failure, and the values of $J_{E S I S}$ and $K \rho_{C}$ at failure. 

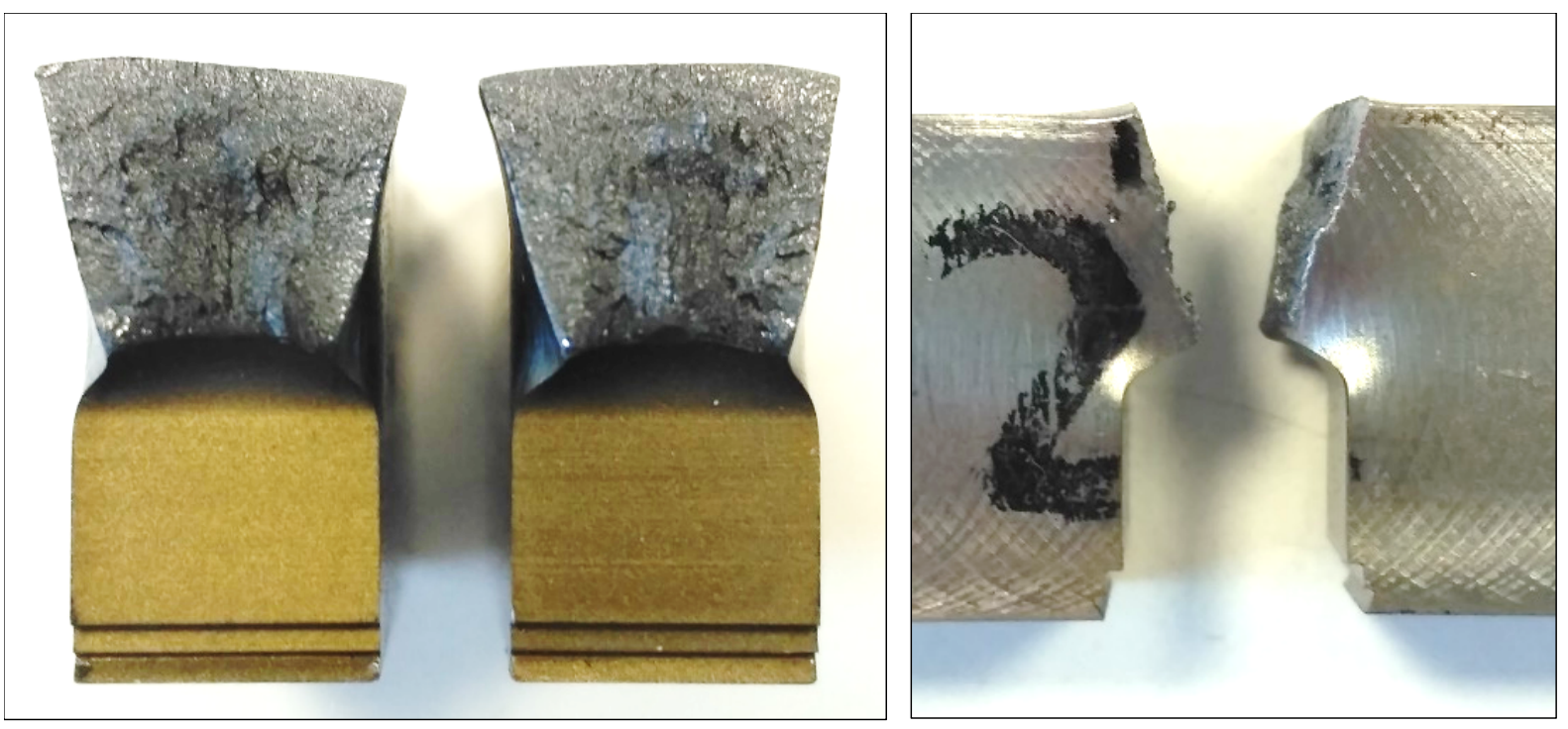

Figure 8: Fracture surface for specimen D26 showing extensive plastic deformation at the free surface but no visible ductile tearing preceding cleavage fracture

Table 5: Experimental Results

\begin{tabular}{|c|c|c|c|c|c|c|c|c|c|c|}
\hline \multirow{2}{*}{$\begin{array}{l}\text { Specimen } \\
\text { ID }\end{array}$} & \multirow{2}{*}{$\begin{array}{c}\mathrm{B} \\
(\mathrm{mm})\end{array}$} & \multirow{2}{*}{$\begin{array}{c}\mathrm{W} \\
(\mathrm{mm})\end{array}$} & \multirow[b]{2}{*}{$\begin{array}{c}\mathrm{a} \\
(\mathrm{mm})\end{array}$} & \multirow[b]{2}{*}{$\stackrel{\rho}{\rho}$} & \multirow[b]{2}{*}{$\mathrm{B} / \mathrm{W}$} & \multirow[b]{2}{*}{$\rho / a$} & \multicolumn{2}{|c|}{ Failure } & \multirow{2}{*}{$\begin{array}{c}J_{E S I S} \\
\left(\mathrm{~kJ} / \mathrm{m}^{2}\right)\end{array}$} & \multirow{2}{*}{$\begin{array}{c}K \rho_{C} \\
(\mathrm{MPa} \sqrt{\mathrm{m}})\end{array}$} \\
\hline & & & & & & & Type & $\begin{array}{l}\text { Load } \\
(\mathrm{kN})\end{array}$ & & \\
\hline D11 & 9 & 18 & 9 & 0.15 & 0.5 & 0.017 & Cleavage & 10.80 & 561 & 364.2 \\
\hline D12 & 9 & 18 & 9 & 0.15 & 0.5 & 0.017 & Cleavage & 11.55 & 1146 & 520.7 \\
\hline D13 & 9 & 18 & 9 & 0.15 & 0.5 & 0.017 & Max Load & 11.13 & 898 & 461.1 \\
\hline D14 & 9 & 18 & 9 & 0.15 & 0.5 & 0.017 & Cleavage & 10.38 & 859 & 450.9 \\
\hline D15 & 9 & 18 & 9 & 0.15 & 0.5 & 0.017 & Max Load & 10.24 & 658 & 394.7 \\
\hline D16 & 9 & 18 & 9 & 0.15 & 0.5 & 0.017 & Cleavage & 9.65 & 506 & 346.0 \\
\hline D21 & 9 & 18 & 9 & 1.20 & 0.5 & 0.133 & Stopped & 12.84 & 2322 & 741.3 \\
\hline D22 & 9 & 18 & 9 & 1.20 & 0.5 & 0.133 & Cleavage & 12.29 & 2196 & 721.0 \\
\hline D23 & 9 & 18 & 9 & 1.20 & 0.5 & 0.133 & Cleavage & 12.08 & 2042 & 695.3 \\
\hline D24 & 9 & 18 & 9 & 1.20 & 0.5 & 0.133 & Max Load & 12.10 & 2852 & 821.6 \\
\hline D25 & 9 & 18 & 9 & 1.20 & 0.5 & 0.133 & Max Load & 11.90 & 1968 & 682.6 \\
\hline D26 & 9 & 18 & 9 & 1.20 & 0.5 & 0.133 & Cleavage & 11.85 & 2009 & 689.5 \\
\hline E11 & 15 & 18 & 9 & 0.15 & 0.833 & 0.017 & Cleavage & 17.04 & 380 & 299.8 \\
\hline E12 & 15 & 18 & 9 & 0.15 & 0.833 & 0.017 & Cleavage & 17.23 & 382 & 300.8 \\
\hline E13 & 15 & 18 & 9 & 0.15 & 0.833 & 0.017 & Cleavage & 17.56 & 412 & 312.1 \\
\hline E14 & 15 & 18 & 9 & 0.15 & 0.833 & 0.017 & Cleavage & 16.31 & 217 & 226.7 \\
\hline E15 & 15 & 18 & 9 & 0.15 & 0.833 & 0.017 & Cleavage & 17.76 & 421 & 315.6 \\
\hline E16 & 15 & 18 & 9 & 0.15 & 0.833 & 0.017 & Cleavage & 16.49 & 278 & 256.6 \\
\hline E21 & 15 & 18 & 9 & 1.20 & 0.833 & 0.133 & Stopped & 19.26 & 1135 & 518.3 \\
\hline E22 & 15 & 18 & 9 & 1.20 & 0.833 & 0.133 & Cleavage & 21.24 & 1565 & 608.6 \\
\hline E23 & 15 & 18 & 9 & 1.20 & 0.833 & 0.133 & Cleavage & 20.11 & 1032 & 494.2 \\
\hline E24 & 15 & 18 & 9 & 1.20 & 0.833 & 0.133 & Cleavage & 21.02 & 1361 & 567.6 \\
\hline E25 & 15 & 18 & 9 & 1.20 & 0.833 & 0.133 & Cleavage & 21.03 & 1528 & 601.4 \\
\hline E26 & 15 & 18 & 9 & 1.20 & 0.833 & 0.133 & Cleavage & 20.96 & 1417 & 579.1 \\
\hline
\end{tabular}


Figure 9(a) presents the experimental results in terms of $K \rho_{C}$ plotted against $B / W . K_{\text {mat }}$ values obtained using the Master Curve (Equation 6) for pre-cracked specimens are also plotted for comparison, with the points corresponding to the median value and the error bars denoting the $5^{\text {th }}$ and $95^{\text {th }}$ percentile values of $K_{\text {mat }}$. Figure 9(a) clearly shows a significant reduction in toughness with increasing specimen thickness for both acute and blunt notches. These data provide clear experimental evidence of the thickness effect that occurs in steel specimens with notches. Figure 9(b) presents the same results as Figure 9(a) but plotted in the same form as Figure 1 with $K \rho_{C} / K_{\text {mat }}$ on the ordinate axis and $\sigma_{N} / \sigma_{0}$ on the abscissa axis, where $\sigma_{N}$ is calculated using Equation 1 . As the thickness of the pre-cracked specimen used to define $K_{\text {mat }}$ is equal to that of the notched specimen, the thickness effect in Figure 9(b) can be attributed to the differences in the extents of out-of-plane constraint loss between the two specimens.

Curves have also been fitted to the test data in Figure 9(b) using Equation 2. These are best-fit curves through the middle of the test data, so the normalising toughness $K_{\text {mat }}$ has also been defined at the median level. If curves were fitted as a lower-bound, for example through the lower $5^{\text {th }}$ percentile of the test data, then the normalising toughness $K_{\text {mat }}$ would also be defined at the lower $5^{\text {th }}$ percentile for consistency. Although the individual $K_{C} / K_{\text {mat }}$ data points would be higher in this case, the fitted $K_{\text {mat }}^{\rho} / K_{\text {mat }}$ curve would be unchanged. As discussed in Section 2, this is one of the useful aspects of the approach: for a given value of $\sigma_{N} / \sigma_{0}$, the ratio $K_{\text {mat }}^{\rho} / K_{\text {mat }}$ remains independent of load, independent of $J$, independent of $\sigma_{w}$, and independent of cleavage fracture probability $P_{f}$. However, the same $P_{f}$ must be used for the definition of both $K_{m a t}^{\rho}$ and $K_{\text {mat }}$ for consistency.
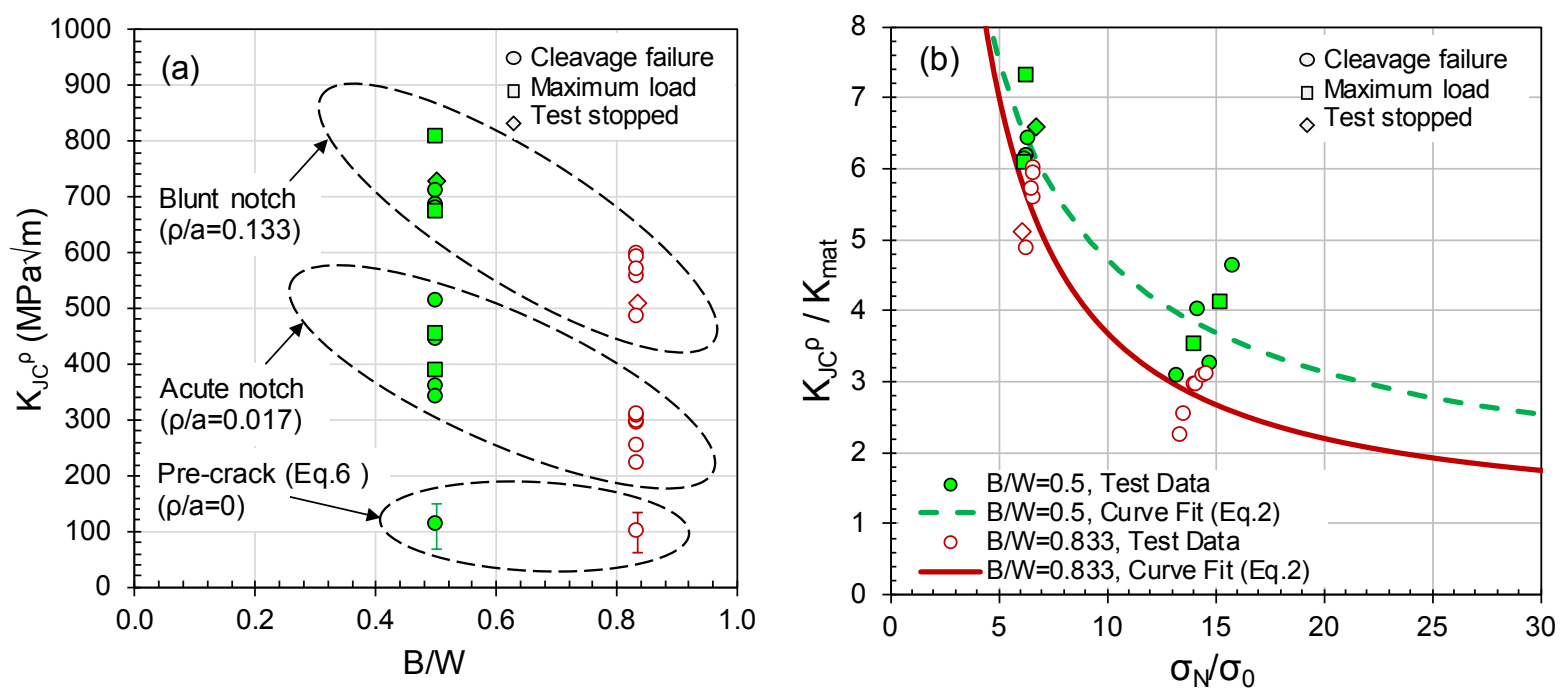

Figure 9: Experimentally measured $K \rho_{C}$ values plotted (a) against specimen thickness $B$, (b) normalised in the form $K \rho_{C} / K_{m a t}$ vs. $\sigma_{N} / \sigma_{0}$.

\subsection{Finite Element Analysis}

Figure 7 compares the load vs. LLD output from the FE analyses with the test data. The FE results correlate well with the test data, with the FE results lying within the scatter of the test data. For the 
acute notch with $\rho / a=0.017$, the FE results slightly over-predict the load at the yield point. A reduction in accuracy of FE models around the yield point has been observed previously [46] for acute notches when modelling materials that exhibit discontinuous yielding with stress-strain curves that retain the Lüders band. The over-prediction of load at the yield point is relatively small in the current case, and as fracture of the test specimens occurs well beyond yield, this is not expected to have a significant impact on the modelling results at failure.

\subsubsection{Calibration of Weibull parameter, $\mathbf{m}$}

As discussed earlier, the thick SENB with acute notch was selected as the high constraint geometry A. All six of these specimens failed by cleavage without prior ductile tearing and are therefore suitable to use for calibration. The thin SENB with blunt notch was selected as the low constraint geometry B, but unfortunately only three of these specimens failed by cleavage without prior ductile tearing. Calibration of the Weibull parameters ideally requires larger datasets - typically ten repeats at each condition - so the size of the available datasets is much smaller than would be preferred. Despite the small calibration datasets, an attempt was made to calibrate the parameters.

To calibrate the Weibull modulus $m$, initial trial values of $m=10,11,12, \ldots, 19$ were chosen. A plot of the error function $R(m)$ vs. $m$ indicated a zero value for the function would be achieved with $m$ slightly below 10. A second iteration of steps $4 \mathrm{a}-4 \mathrm{c}$ from Section 4.3.1 of the paper was therefore carried out for $\mathrm{m}=9.0,9.1,9.2, \ldots, 9.9$. A plot of $R(m)$ vs. $m$ showed the error function was close to zero at $m=9.1$. This was taken to be the 'calibrated' value of $m$. Applying step 5 of the procedure resulted in different values of $\sigma_{u} / \sigma_{0}$ for each specimen thickness modelled, and these values are summarised in Table 6.

Table 6: Calibrated Weibull parameters for different thicknesses

\begin{tabular}{|c|c|c|}
\hline $\mathbf{B} / \mathbf{W}$ & $\boldsymbol{m}$ & $\boldsymbol{\sigma}_{\boldsymbol{u}} / \boldsymbol{\sigma}_{\mathbf{0}}$ \\
\hline 0.5 & \multirow{4}{*}{9.1} & 3.47 \\
\hline 0.833 & & 3.67 \\
\cline { 1 - 1 } & & 3.74 \\
\hline 1.0 & & 3.91 \\
\hline 1.5 &
\end{tabular}

\subsubsection{Failure predictions}

Figure 10 compares the predicted values of $P_{f}$, calculated using Equation 12, with the values of $\sigma_{w} /$ $\sigma_{0}$ at failure. Predictions for the thinner specimens with $B / W=0.5$ are shown in Figure 10(a) and those for the thicker specimens with $B / W=0.833$ are shown in Figure 10(b). The Weibull stress model tends to over-predict the scatter when using the standard $K_{\min }$ value of $20 \mathrm{MPa} \sqrt{\mathrm{m}}$ specified for precracked specimens in ASTM E1921. The use of a higher $K_{\min }$ value reduces the spread of the predictions and brings them into closer alignment with the test data, however the use of a higher value also raises the question of the most appropriate value to select for $K_{\min }$ when assessing notched specimens (the value of $50 \mathrm{MPa} \sqrt{\mathrm{m}}$ is an arbitrarily selected value to show the sensitivity of the approach to this value). It is therefore convenient to consider the results at $P_{f}=0.632$, where the predictions are insensitive to the value assumed for $K_{\min }$ and hence insensitive to $\sigma_{w, \min }$.

The datasets used for calibration are shown as solid black circles, so the predictive capability of the method can be judged by comparing the prediction with the open circles. The predictions for the thin specimens match very well with the acute notch test data Figure 10(a), however for the thicker 
specimens the model under-predicts failure of the blunt notch specimens Figure 10(b). Given the relatively small datasets used for calibration of $m$, the close correlation between predictions and test data for the thin specimens in Figure 10(a) is perhaps more surprising than the under-prediction of the blunt specimen in Figure 10(b).
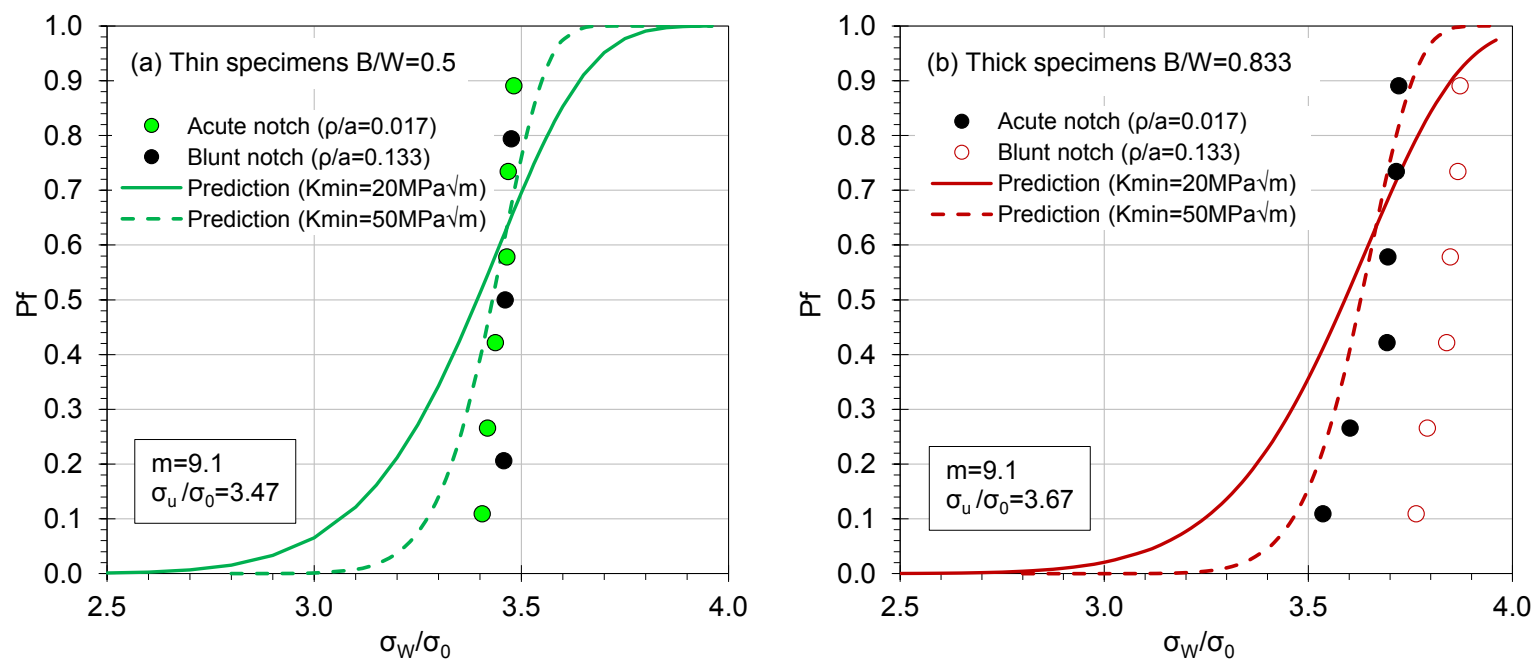

Figure 10: Failure predictions for (a) thin specimens with $B / W=0.5$, (b) thick specimens with $B / W=0.833$

Figure 11(a) compares the predicted values of $K \rho_{C}$ with the test data, plotted against $B / W . K_{\text {mat }}$ values obtained using the Master Curve (Equation 6) for pre-cracked specimens are also plotted for comparison at $P_{f}=0.632$. The predictions, which were made at the two discrete $B / W$ values of 0.5 and 0.833 , have been joined together with straight black lines for the purposes of clarity, although in reality the trend is unlikely to be linear. The predictions would be expected to pass close to the centre of the experimental data; although they are reasonable for the thinner specimens, there is a tendency to under-predict $K \rho_{C}$ for the thicker, blunter notch, which is consistent with Figure 10(b). This same trend is also noticeable in Figure 11(b), which shows the same test results and predictions but plotted in a form consistent with Figure 1. Despite this under-prediction, the overall general trend of the variation of $K \rho_{C}$ with $B / W$ appears broadly reasonable considering the reduction in accuracy expected due to the limited data that was available for calibration. 

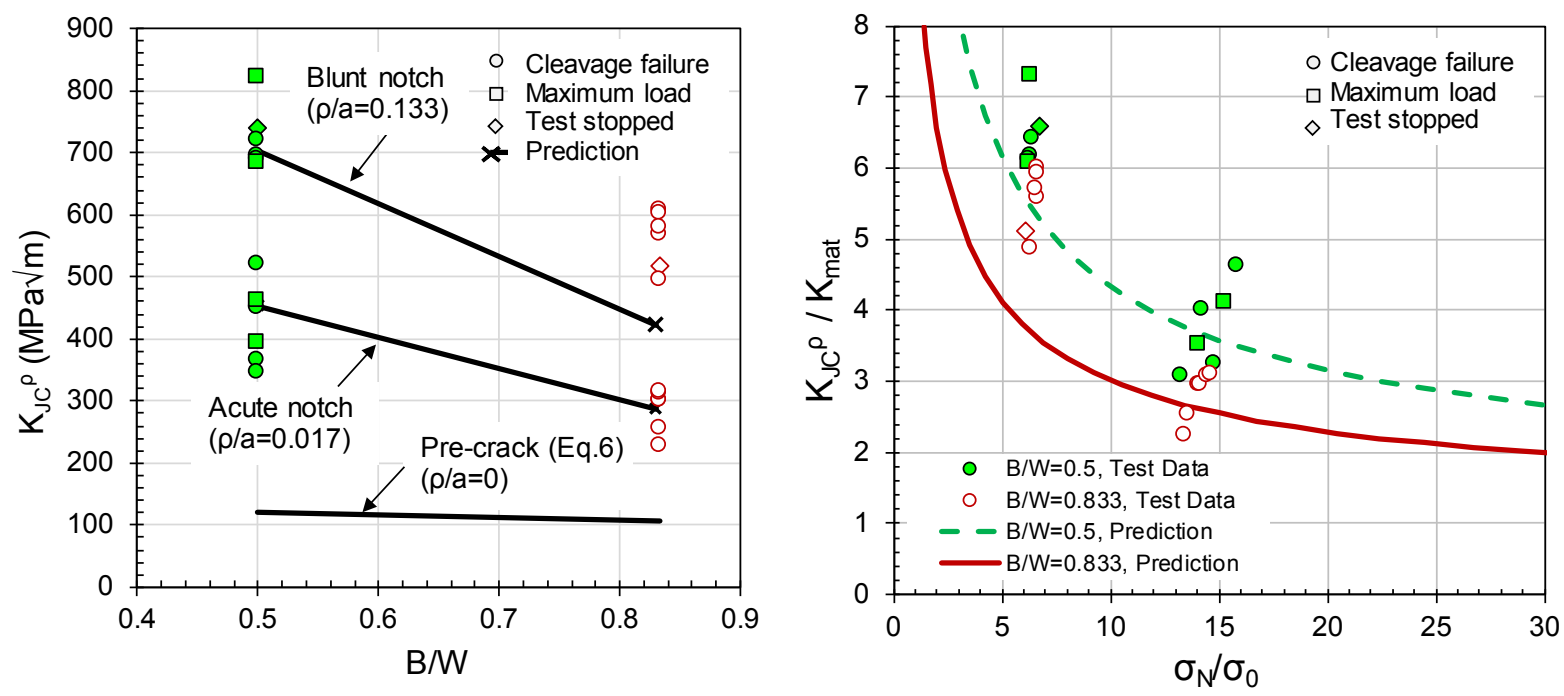

Figure 11: Failure predictions (a) plotted against $B / W$, (b) normalised in the form $K \rho_{C} / K_{\text {mat }}$ vs. $\sigma_{N} / \sigma_{0}$

\subsubsection{Toughness scaling to other thicknesses}

Toughness scaling predictions for SENB specimens with thicknesses greater than those in the experimental programme are presented in Figure 12. The toughness scaling diagram for the blunt notch radius with $\rho / a=0.133$ is shown in Figure 12(a), plotted in terms of $P_{f}$ on the ordinate axis calculated using Equation 12 with $K_{m i n}=20 \mathrm{MPa} \sqrt{ } \mathrm{m}$, and $J$ on the abscissa axis calculated using Equation 7. Predicted values of effective notch toughness are plotted in Figure 12(b) as a function of $B / W$, where the predictions correspond to $P_{f}=0.632$ for consistency with the results presented in Figure 11(a). The FE predictions indicate that for both notch radii, the effective notch toughness becomes relatively insensitive to thickness above $B / W=0.833$. The test data are in agreement for the acute notch, but as the FE under-predicts $K \rho_{C}$ for the blunt notch, it could be argued that the insensitive region starts closer to $B / W=1.0$. 

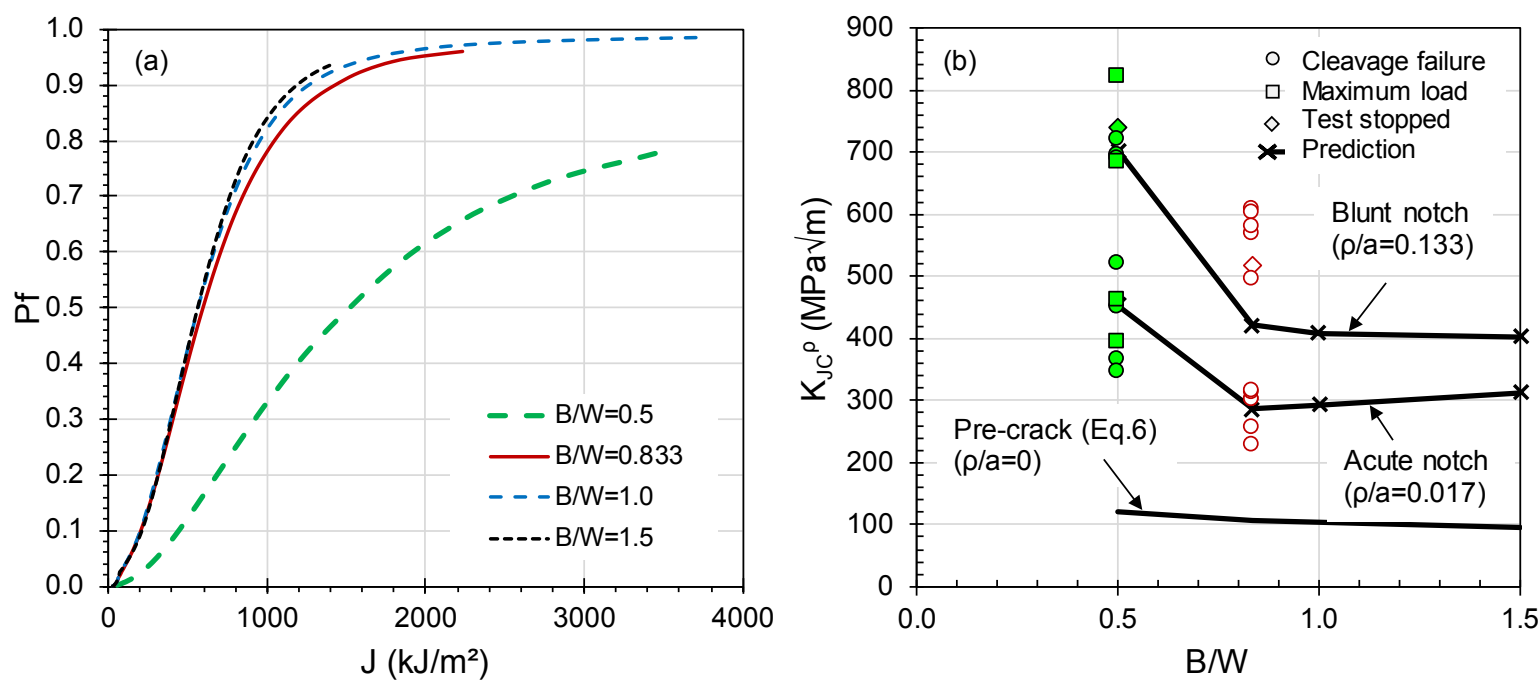

Figure 12: FE predictions for thicknesses up to $B / W=1.5$, (a) toughness scaling diagram for $\rho / a=0.133$, (b) predictions for both notch radii plotted against $B / W$ 


\section{DISCUSSION}

The effect of thickness on effective notch toughness has previously been discussed in [47]. The magnitude of the effect was predicted using Weibull stress analysis in [23], and the test data in this paper provide clear experimental evidence of this effect.

When values of $K \rho_{C}$ are plotted without being normalised by $K_{\text {mat }}$, such as in Figure 9(a) and Figure 11(a), the observed thickness effect is due to a combination of two distinct components: primarily a mechanical out-of-plane constraint loss effect due to the loss of plane strain conditions that occurs with decreasing thickness; and to a lesser degree a microstructural weakest link effect which is relevant for cleavage fracture. The latter effect arises due a shorter crack front length and hence smaller plastic zone volume decreasing the probability of sampling a microstructural feature capable of triggering cleavage fracture, compared with a thick specimen. Normalising the values of $K \rho_{C}$ by the corresponding fracture toughness $K_{m a t}$ for a pre-cracked specimen of the same thickness, e.g. Figure 9(b) and Figure 11(b), results in plots that show only the mechanical constraint loss effect.

The overall toughness benefit defined by $K_{\text {mat }}^{\rho} / K_{\text {mat }}$ therefore arises due to a combination of the inplane effect of the notch radius and the out-of-plane constraint loss which itself is enhanced by the presence of the notch radius. Both the test data and the FE predictions indicate that for the thin SENB specimens with $B / W=0.5$, the out-of-plane constraint loss is as significant as the in-plane effect of the notch radius alone. This is consistent with the numerical study of Compact Tension (CT) specimens in [23] which showed the out-of-plane constraint loss effect was as significant as the in-plane notch effect for CT specimens with $B / W=0.5$.

Fracture toughness testing standards such as [38,39] provide minimum thickness requirements to ensure that the out-of-plane constraint loss is minimised in pre-cracked test specimens, for example [39]:

$$
B \geq \frac{100 \mathrm{~J}}{\sigma_{y}}
$$

where $J$ is defined for a pre-cracked specimen. Equation 13 was used to design the test programme in this paper, specifically to select the thinnest possible specimen that would still meet this criterion if the specimen was pre-cracked instead of notched. Using the Master Curve to define median fracture toughness properties, Equations 6 and 13 were solved for a range of trial thickness values to find the lowest integer value of $B$ that would satisfy Equation 13, and hence meet the standard criterion to ensure plane strain conditions for a pre-cracked specimen. This resulted in $B=9 \mathrm{~mm}$ (i.e. $B / W=0.5$ ) being chosen for the thinnest specimen in the test programme. In contrast, for the test specimens containing notches, the results in Figure 12(b) indicate that a minimum thickness closer to $B / W=1.0$ is required to minimise out-of-plane constraint loss. This finding is consistent with the numerical analysis in [23] which showed that although significant out-of-plane constraint loss occurred in CT specimens with $B / W=0.5$, doubling the thickness to $B / W=1.0$ was sufficient to eliminate the out-ofplane constraint loss. It is important to note that halving the width $W$ instead of doubling the thickness $B$ to achieve the same ratio $B / W=1.0$ would not achieve the same result; not only would the specimen still be affected by out-of-plane constraint loss, but the reduced $W$ may also lead to in-plane constraint loss. It is therefore important to note that a specific $B / W$ ratio should not be regarded as a universal criterion for eliminating out-of-plane constraint loss, the ratio has used in this paper only as a convenient normalised measure of specimen thickness. Any universal criterion for defining the 
minimum specimen thickness to ensure plane strain conditions would be in the form of a modification to Equation 13 , rather than a single $B / W$ ratio.

The philosophy adopted in BS7910 [2] is to measure fracture toughness using full thickness test specimens, i.e. specimens with a thickness $B$ equal to the thickness of the structure being assessed. Although this is appropriate for assessing cracked structures using pre-cracked test specimens which meet the minimum thickness requirements, test specimens containing notches are more likely to suffer from out-of-plane constraint loss than pre-cracked specimens of the same thickness. Therefore, even full-thickness test specimens may exhibit higher toughness than would be expected if conditions were fully plane strain. Using such a value of $K_{\text {mat }}^{\rho}$ would be non-conservative in an NFAD assessment if the non-sharp defect in the structure being assessed was in plane strain, for example the deepest part of a long surface-breaking notch.

\section{CONCLUSIONS}

The main conclusions of this work are as follows:

- The test data in this paper provide clear experimental evidence of a significant thickness effect on the effective cleavage toughness $K_{\text {mat }}^{\rho}$ measured using SENB specimens containing a U-notch instead of a pre-crack. This effect is over and above the microstructural weakest link effect arising from differences in the volume of the plastic zone.

- The toughness benefit due to the notch, $K_{\text {mat }}^{\rho} / K_{\text {mat }}$, is a function of both the in-plane effect of the notch radius and an out-of-plane constraint loss which itself is enhanced by the presence of the notch radius. The test data and FE modelling results indicate that the effect of this out-of-plane constraint loss on $K_{m a t}^{\rho} / K_{m a t}$ can be of the same order of magnitude as the in-plane effect of the notch radius alone.

- For the material considered in this paper, significant out-of-plane constraint loss occurred for notched specimens with $B / W=0.5$, a geometry that if pre-cracked would have met the minimum thickness requirement mandated by ASTM E1820.

- Doubling the thickness to $B / W=1.0$ was sufficient to eliminate the out-of-plane constraint loss for the material and geometry considered, an observation consistent with a previous numerical study [23]. $K_{\text {mat }}^{\rho}$ was relatively insensitive to thickness for $B / W>1.0$.

- The use of experimentally measured $K_{\text {mat }}^{\rho}$ values in an NFAD assessment of a structure may be non-conservative if $B / W<1.0$, due to the loss of plane strain conditions in the test specimen.

\section{ACKNOWLEDGEMENTS}

This paper is published with the permission of Wood, the University of Cantabria, and the UK Atomic Energy Authority. The authors of this work would like to express their gratitude for financial support from: 
- The Spanish Ministry of Science and Innovation, under project MAT2014-58443-P: “Análisis del comportamiento en fractura de componentes estructurales con defectos en condiciones debajo confinamiento tensional", on which the results of this paper are based;

- Wood, via a contribution to the R6 Development Programme;

- The Research Councils UK (RCUK) Energy Programme [grant number EP/P012450/1]. To obtain further information on the data and models underlying this paper please contact: PublicationsManager@ukaea.ac.uk.

\section{REFERENCES}

1. R6 - Revision 4: assessment of the integrity of structures containing defects, Amendment 11: March 2015. EDF Energy, Gloucester, UK.

2. BSI. BS7910:2013+A1:2015, incorporating corrigenda Nos. 1 and 2, 2016: Guide on methods for assessing the integrity of flaws in metallic structures. British Standards Institution, 2016.

3. API 579-1/ASME FFS-1, Fitness-for-Service, jointly published by the American Petroleum Institute and the American Society for Mechanical Engineers, June 2016.

4. BSI. BS 7448-1: fracture mechanics toughness tests - Part 1: method for determination of KIC, critical CTOD and critical J values of metallic materials. British Standards Institution; 1999.

5. ASTM E1820-11. Standard Test Method for Measurement of Fracture Toughness. ASTM, 2011.

6. ESIS. ESIS P2-92: ESIS procedure for determining the fracture behaviour of materials; 1992.

7. Wang WQ, Li AJ, Li PN, Ju DY. Engineering approach for notch elastic-plastic fracture analysis. International Journal of Pressure Vessels and Piping 1994; 60:1-16.

8. Smith E. Fracture initiation at the root of a blunt flaw: description in terms of $\mathrm{Kr}-\mathrm{Lr}$ failure assessment curves. International Journal of Pressure Vessels and Piping 1999;76(11):799-800.

9. Matvienko YG. Local fracture criterion to describe failure assessment diagrams for a body with a crack/notch. International Journal of Fracture 2003;124:107-12.

10. Cicero S, Gutierrez-Solana F, Horn AJ. Experimental analysis of differences in mechanical behaviour of cracked and notched specimens in a ferritic-pearlitic steel: Considerations about the notch effect on structural integrity. Engineering Failure Analysis 2009;16:2450-66.

11. Cicero S, Gutiérrez-Solana F, Álvarez JA. Structural integrity assessment of components subjected to low constraint conditions. Engineering Fracture Mechanics 2008;75(10):3038-59.

12. Horn AJ, Sherry AH. An engineering assessment methodology for non-sharp defects in steel structures - Part I: procedure development. International Journal of Pressure Vessels and Piping 2012;89:137-50.

13. Han J-J, Larossa NO, Kim Y-J, Ainsworth RA. Blunt defect assessment in the framework of the failure assessment diagram. International Journal of Pressure Vessels and Piping 2016; 146:39-54.

14. Horn AJ, Aird C. Limits of Applicability of the Notch Failure Assessment Diagram. Proceedings of the ASME 2018 Pressure Vessels and Piping Conference, July 15-20, 2018, Prague, Czech Republic. PVP2018-85062.

15. Horn AJ, Budden PJ. Development of test guidance for single edge notch bend fracture toughness specimens containing notches instead of fatigue pre-cracks. Proceedings of the ASME 2015 Pressure Vessels and Piping Conference, July 19-23, 2015, Boston, Massachusetts, USA. PVP2015-45474.

16. Horn AJ, Budden PJ. Development of test guidance for compact tension fracture toughness specimens containing notches instead of fatigue pre-cracks. Proceedings of the $23^{\text {rd }}$ Conference on Structural Mechanics in Reactor Technology (SMiRT-23), Manchester, UK, August 10-14, 2015. Paper no. 287.

17. Cicero S, Garcia T, Madrazo V, Cuervo J, Ruiz E, Gutierrez-Solana F. Analysing the Notch Effect within the Ductile-to-Brittle Transition Zone of S275JR Steel. Proceedings of the ASME 2013 Pressure Vessels and Piping Conference, July 14-18, 2013, Paris, France. PVP2013-97017.

18. Cicero S, Madrazo V, Garcia T, Cuervo J, Ruiz E. On the Notch Effect in Load Bearing Capacity, Apparent Fracture Toughness and Fracture Mechanisms of Polymer PMMA, Aluminium Alloy 
A17075-T651 and Structural Steels S275JR and S355J2. Engineering Failure Analysis 29 (2013) 108-121.

19. Cicero S, Gutierrez-Solana F, Alvarez JA. Structural Integrity Assessment of Components Subjected to Low Constraint Conditions. Engineering Fracture Mechanics 75 (2008) 3038-3059.

20. Landes JD, Begley JA. Experimental Methods for Elastic-Plastic and Post-Yield Fracture Toughness Measurements, in Post-yield Fracture Mechanics, D.G.H. Latzko, Editor. 1979, Applied Science Publishers Ltd, London. p. 211-253.

21. Livieri P. Use of J-integral to Predict Static Failures in Sharp V-notches and Rounded U-Notches. Engineering Fracture Mechanics 75 (2008) 1779-1793.

22. Barati E, Berto F. Fracture Assessment of U-notches Under Mode I Loading By Means of Critical Value of the J-integral. Procedia Engineering 10 (2011) 807-812

23. Horn AJ, Sherry AH, Budden PJ. Size and geometry effects in notched compact tension specimens. International Journal of Pressure Vessels and Piping 2017;154:29-40.

24. Matvienko, YG. The effect of out-of-plane constraint in terms of the T-stress in connection with specimen thickness. Theoretical and Applied Fracture Mechanics 2015;80:49-56.

25. Liu Z, Wang X, Tang J, Deng C, Zhao H, Chen X. The effects of in-plane and out-of-plane constraints on J-R curves for X80 steel: A study using clamped SENT specimens. Engineering Fracture Mechanics 2019;206:342-358.

26. Horn AJ, Sherry AH. Prediction of cleavage fracture from non-sharp defects using the Weibull stress based toughness scaling model. International Journal of Pressure Vessels and Piping 2010;87:670-80.

27. Shin CS. A discussion on various estimations of elastic stress distributions and stress concentration factors for sharp edge notches. International Journal of Fatigue, 1986. 8(4): 235-237

28. Creager M, Paris PC. Elastic field equations for blunt cracks with reference to stress corrosion cracking. International Journal of Fracture Mechanics, 1967. 3: p. 247-252

29. Kim JS, Larrosa NO, Horn AJ, Kim YJ, Ainsworth RA. Notch Bluntness Effects on Fracture Toughness of a Modified S690 Steel at $150^{\circ}$ C. Engineering Fracture Mechanics 2018;188:250267.

30. Beremin FM. A Local Criterion for Cleavage Fracture of a Nuclear Pressure Vessel Steel. Metallurgical Transactions A 1983;14A,2277-2287.

31. Gao X, Ruggieri C, Dodds Jr RH. Calibration of Weibull Stress Parameters using Fracture Toughness Data. International Journal of Fracture 1998;92(2),175-200.

32. EN 10025-4:2004, Technical delivery conditions for thermomechanical rolled weldable fine grain structural steels, European Committee for Standardization, 2004.

33. Cicero, S., García, T., Madrazo, V. Application and validation of the Notch Master Curve in medium and high strength structural steels, Journal of Mechanical Science and Technology, 2015;29:4129-4142.

34. Cicero, S., García, T., Madrazo, V. Structural integrity analysis of notched ferritic steels operating within their ductile-to-brittle transition zone: An approach from Failure Assessment Diagrams and the Notch Master Curve, Engineering Failure Analysis, 2015;58:134-148.

35. ASTM E8-E8M-13a, Standard test methods for tension testing of metallic materials, American Society for Testing and Materials, Philadelphia, USA, 2013.

36. Ingham T., Knee N., Milne I., Morland E. Fracture toughness in the transition regime for A533B-1 steel: prediction of large specimen results from specimen tests. ASTM STP 1020, 1989. p.369-389.

37. K. Wallin, The size effect in $\mathrm{K}_{\mathrm{Ic}}$ results, Engineering Fracture Mechanics, 1985;22:149-163.

38. ASTM E1921-13a, Test method for the determination of reference temperature $T_{0}$ for ferritic steels in the transition range, American Society for Testing and Materials, Philadelphia, USA, 2013

39. ASTM E1820-13. Standard test method for measurement of fracture toughness, American Society for Testing and Materials, Philadelphia, 2013.

40. ESIS P2-92. ESIS Procedure for Determining the Fracture Behaviour of Materials. ESIS, 1992.

41. ABAQUS 6.12. ABAQUS Documentation, Dassault Systemes, Providence, RI, USA. 
42. Bilby BA, Cardew GE, Goldthorpe MR, Howard IC. A finite element investigation of the effect of specimen geometry on the fields of stress and strain at the tips of stationary cracks, in size effects in fracture. Mechanical Engineering Publications; 1986:37-46.

43. Williams ML. Stress singularities resulting from various boundary conditions in angular corners of plates in extension. Journal of Applied Mechanics 1952;19:526-528.

44. Gao X, Ruggieri C, Dodds Jr RH. Calibration of Weibull stress parameters using fracture toughness data. International Journal of Fracture 1998;92(2):175-200.

45. Ruggieri C, Dodds Jr RH. A transferability model for brittle fracture including constraint and ductile tearing effects: a probabilistic approach. International Journal of Fracture 1996;79:309340

46. Horn AJ, Cicero S, Bannister A, Budden P. Development of Guidance for the Assessment of Non-Sharp Defects using the Notch Failure Assessment Diagram. Proceedings of the ASME 2016 Pressure Vessels and Piping Conference, July 17-21, 2016, Vancouver, British Columbia, Canada. PVP2016-63537.

47. Taylor D. The Theory of Critical Distances: a New Perspective in Fracture Mechanics, 2007. Elsevier, Oxford, UK. 
Dr. Anthony Horn: Conceptualisation, Methodology, Software, Formal analysis, Investigation, Resources, Writing - Original Draft, Visualisation, Project administration, Funding acquisition

Prof. Sergio Cicero: Conceptualisation, Methodology, Formal analysis, Investigation, Resources, Writing - Original Draft, Visualisation, Supervision, Project administration, Funding acquisition

Dr. David Andres: Investigation, Writing - Review and Editing 